\title{
Higher spin entanglement entropy
}

\section{Jiang Long}

Department of Physics and State Key Laboratory of Nuclear Physics and Technology, Peking University, No. 5 Yiheyuan Rd, Beijing 100871, P.R. China

E-mail: 1j301@pku.edu.cn

ABSTRACT: In this paper, we develop a perturbation formulation to calculate the single interval higher spin Rényi and entanglement entropy for two dimensional conformal field theory with $\mathcal{W}_{\infty}(\lambda)$ symmetry. The system is at finite temperature and is deformed by higher spin chemical potential. We manage to compute higher spin Rényi entropy with various spin deformations up to order $\mathcal{O}\left(\mu^{2}\right)$. For spin 3 deformation, we calculate exact higher spin Rényi and entanglement entropy up to $\mathcal{O}\left(\mu^{4}\right)$. When $\lambda=3$, in the large $c$ limit, we find perfect match with tree level holographic higher spin entanglement entropy up to order $\mu^{4}$ obtained by the Wilson line prescription. We also find quantum corrections to higher spin entanglement entropy which is beyond tree level holographic results. The quantum correction is universal at order $\mu^{4}$ in the sense that it is independent of $\lambda$. Our computation relies on a multi-valued conformal map from $n$-sheeted Riemann surface $\mathcal{R}_{n}$ to complex plane and correlation functions of primary fields on complex plane. The method can be applied to general conformal field theories with $\mathcal{W}$ symmetry.

Keywords: Higher Spin Gravity, Higher Spin Symmetry, Wilson, 't Hooft and Polyakov loops, Chern-Simons Theories

ArXiv EPRINT: 1408.1298 


\section{Contents}

1 Introduction 2

2 Rényi and entanglement entropy $\quad 3$

$\begin{array}{llll}3 & \mathrm{AdS}_{3} & \text { higher spin gravity } & 5\end{array}$

3.1 Asymptotic symmetry and $\mathcal{W}$ algebra 6

$\begin{array}{lll}3.2 & \text { Higher spin black hole } & 7\end{array}$

4 Holographic HSEE $\quad 9$

5 Partition function of higher spin black hole $\quad 11$

$6 \quad$ HSRE and HSEE

$\begin{array}{lll}6.1 \mathcal{O}\left(\mu^{2}\right) \text { correction } & 13\end{array}$

$\begin{array}{lll}6.2 \mathcal{O}\left(\mu^{3}\right) \text { correction } & 15\end{array}$

$\begin{array}{lll}6.3 \mathcal{O}\left(\mu^{4}\right) \text { correction } & 15\end{array}$

$\begin{array}{lll}6.3 .1 & \mathcal{O}(c) & 16\end{array}$

$\begin{array}{lll}\text { 6.3.2 Quantum correction } & 18\end{array}$

$\begin{array}{lll}6.3 .3 & \mathcal{W}_{\infty}(\lambda) \text { theory } & 19\end{array}$

$\begin{array}{llr}7 & \text { Conclusion and discussion } & 20\end{array}$

A Correlation function on $\mathcal{R}_{n} \quad 22$

$\begin{array}{lll}\text { A.1 Two point function on } \mathcal{R}_{n} & 22\end{array}$

A.2 Three point function on $\mathcal{R}_{n} \quad 24$

$\begin{array}{lll}\text { A.3 } & \text { Four point function on } \mathcal{R}_{n} & 24\end{array}$

A.4 Higher point function on $\mathcal{R}_{n}$

B Integrals $\quad 33$

B.1 Integral $F[J, j] \quad 35$

B.2 Integral in $\mathcal{O}\left(\mu^{4}\right) \quad 38$

C $\mathcal{O}\left(\mu^{2}\right)$ correction to Rényi entropy and entanglement entropy of other $\begin{array}{ll}\text { spins } & 40\end{array}$

D Quantum correction of partition function of higher spin black hole $\quad 41$ 


\section{Introduction}

Entanglement entropy and its generalization Rényi entropy are important quantities to study quantum systems. Entanglement entropy is a good parameter to characterize the effective degree of freedom of a region which is entangled with the rest of the system. It has interesting applications in condensed matter systems [3-5]. In general, they are hard to compute. Surprisingly, inspired by AdS/CFT correspondence, Ryu and Takayanagi [46] proposed that entanglement entropy in a conformal field theory could be calculated from a minimal surface in the dual bulk. Their beautiful work provides an effective way to compute entanglement entropy. At the same time, it opens a new window to study $A d S / C F T$, especially the emergence of spacetime in gravity.

On the other side, another important development in $A d S / C F T$ in recent years is the higher spin holography [6-8], which suggests explicit duality between Vasiliev higher spin theory $[9,10]$ and vectorial conformal field theory in the large $N$ limit. Among various proposals, $H S / C F T_{2}$ [11], which relates higher spin theory in $A d S_{3}$ [12] to $\mathcal{W}_{N}$ minimal model in large $N$ limit, is of particular interest. This duality triggered various studies in $A d S_{3}$ gravity and CFT with $\mathcal{W}$ symmetry, please find nice reviews $[13,14]$ on this topic.

Higher spin Rényi entropy (HSRE) and higher spin entanglement entropy(HSEE) arise from the combination of the previous two separate branches. In the field theory side, when there are higher spin deformations, the partition function can be written schematically as

$$
Z=\left\langle\exp -\mu \int W-\bar{\mu} \int \bar{W}\right\rangle,
$$

where $\mu(\bar{\mu})$ is the chemical potential, $W(\bar{W})$ is the corresponding higher spin current. It would be interesting to consider the deformation of entanglement entropy in this case. In the bulk, as the holographic entanglement entropy relates to a geometric object in usual Einstein-Hilbert theory, a generalization to holographic HSEE may provide insights on mysterious higher spin geometry. Also, they are expected to provide non-trivial check of $H S / C F T$ correspondence. Several works on this issue have been done. In $[15,16]$, for the theory with $\mathcal{W}$ symmetry, two interval Rényi entropy without classical higher spin deformation has been calculated in the short interval limit, the quantum one-loop results in the gravity and CFT side match exactly up to $\mathcal{O}\left(x^{8}\right)$, where $x$ is the cross ratio constructed from the two intervals. When there are higher spin chemical potential deformations, one interesting configuration ${ }^{1}$ is the higher spin black hole [23]. In these cases, a holographic HSEE has been proposed by Wilson line prescription [32, 47]. It has been checked up to $\mathcal{O}\left(\mu^{2}\right)$ for the CFT with $\mathcal{W}_{3}$ symmetry $[44,48]$. Other works related to holographic HSEE can be found in [45].

In this paper, we develop a general prescription to calculate single interval HSRE and HSEE from CFT side perturbatively. Instead of inserting twist operators, we use a multivalued conformal map from a $n$-sheeted Riemann surface to a complex plane. For spin 3 deformation, we find the HSRE and HSEE of $\mathcal{W}_{\infty}(\lambda)$ theory up to $\mathcal{O}\left(\mu^{4}\right)$. We show that the $\mathcal{O}\left(\mu^{2}\right)$ correction of HSRE and HSEE are indeed universal, in the sense that they are

\footnotetext{
${ }^{1}$ There are other configurations, such as chiral deformation solution [24, 25].
} 
independent of the value of $\lambda$ up to a normalization constant. At the $\mathcal{O}\left(\mu^{4}\right)$, we find the classical and all loop quantum corrections to HSRE and HSEE. For $\lambda=3$, the classical part matches with the gravity result exactly. Our method is sufficiently general to extend to all kinds of higher spin deformations.

The structure of this paper is as follows. In section 2, we review the general method to calculate $\mathrm{N}$ interval Rényi entropy and entanglement entropy in two dimensional conformal field theory, emphasizing the importance of a conformal map from $n$-sheeted Riemann surface to complex plane and its multi-valued property. In section 3 , we review pure higher spin gravity in $\mathrm{AdS}_{3}$, including its asymptotic symmetry, higher spin black hole and its partition function. Some important results on $\mathcal{W}$ symmetry and black hole partition function are shown at the same time. In section 4, we briefly review the holographic HSEE and expand the results of $\operatorname{sl}(3)$ theory up to $\mathcal{O}\left(\mu^{4}\right)$. In section 5 , we calculate perturbative partition function of higher spin black hole, which is a good exercise for HSRE and HSEE. In section 6, we calculate HSRE and HSEE up to $\mathcal{O}\left(\mu^{4}\right)$ and match HSEE to holographic results in section 4 . Discussion and conclusion are included in the last section. Some technical details are collected in four appendices.

\section{Rényi and entanglement entropy}

To define entanglement entropy, we suppose the system has a density matrix $\rho$ and then divide the system into a subsystem $\mathrm{A}$ and its complement $\mathrm{B}$. The total Hilbert space is factorized into $\mathcal{H}_{A} \otimes \mathcal{H}_{B}$. We trace out the information of $\mathrm{B}$ and obtain the reduced density matrix of $\mathrm{A}$,

$$
\rho_{A}=\operatorname{tr}_{B} \rho,
$$

then the entanglement entropy of A is defined as the standard von Neumann entropy,

$$
S_{A}=-t r_{A} \rho_{A} \log \rho_{A}
$$

A useful notion associated to the entanglement entropy is the $n$-th Rényi entropy $S^{(n)}$,

$$
S_{A}^{(n)}=\frac{1}{1-n} \log \operatorname{tr} \rho_{A}^{n}=\frac{\log Z_{n}-n \log Z_{1}}{1-n} .
$$

By analytical continuation of $n \rightarrow 1$, we find the entanglement entropy,

$$
S_{A}=\lim _{n \rightarrow 1} S_{A}^{(n)} .
$$

The procedure by introducing Rényi entropy to find the entanglement entropy is called the replica trick [1].

In two dimensions, the replica trick is applied as follows. Suppose the original CFT is defined on a Riemann surface $\mathcal{R}_{1}$. We uplift the theory to $n$ disconnected sheets and there is a branch cut along $A$ in each sheet. We glue the branch cut successively and find an

\footnotetext{
${ }^{2}$ Usually, this is the complex plane $\mathcal{C}$ or those can be conformally transformed to the complex plane. Indeed, in this paper, we will consider the complex plane or the finite temperature version, say, a cylinder.
} 
$n$-sheeted Riemann surface $\mathcal{R}_{n}$. The partition function in the $n$-sheeted Riemann surface is denoted as $Z_{n}$.

In general, the subsystem $A$ consists of $N$ intervals,

$$
A=\left\{\omega \mid \operatorname{Im} \omega=0, \operatorname{Re} \omega \in\left[u_{1}, v_{1}\right] \cup \cdots \cup\left[u_{N}, v_{N}\right]\right\} .
$$

The partition function ${ }^{3} Z_{n, N}$ could be determined by introducing primary twist(and antitwist) operators [2] with conformal dimension $h_{n}=\bar{h}_{n}=\frac{c}{24}\left(n-\frac{1}{n}\right)$ at each point $u_{i}\left(v_{i}\right)$, such that $Z_{n, N}$ is a $2 n$ point function of a $\mathbb{Z}_{n}$ cyclic orbifold conformal field theory on $\mathcal{R}_{1}$,

$$
Z_{n, N}=\langle 1\rangle_{\mathcal{R}_{n, N}}=\left\langle\sigma_{n}\left(u_{1}\right) \tilde{\sigma}_{n}\left(v_{1}\right) \cdots \sigma_{n}\left(u_{N}\right) \tilde{\sigma}_{n}\left(v_{N}\right)\right\rangle_{\mathcal{R}_{1}} .
$$

The expectation value of arbitrary operator $\mathcal{O}$ on $\mathcal{R}_{n, N}$ is

$$
\langle\mathcal{O}(z)\rangle_{\mathcal{R}_{n, N}}=\frac{\left\langle\mathcal{O}(z) \sigma_{n}\left(u_{1}\right) \tilde{\sigma}_{n}\left(v_{1}\right) \cdots \sigma_{n}\left(u_{N}\right) \tilde{\sigma}_{n}\left(v_{N}\right)\right\rangle_{\mathcal{R}_{1}}}{\left\langle\sigma_{n}\left(u_{1}\right) \tilde{\sigma}_{n}\left(v_{1}\right) \cdots \sigma_{n}\left(u_{N}\right) \tilde{\sigma}_{n}\left(v_{N}\right)\right\rangle_{\mathcal{R}_{1}}}
$$

For the single interval, suppose the original CFT is defined on a complex plane $\mathcal{C}$, there is a direct conformal transformation which maps the $n$-sheeted Riemann surface $\mathcal{R}_{n, 1}$ to the complex plane,

$$
\omega^{n}=\frac{z-u}{z-v}
$$

where $z$ and $\omega$ are the coordinates defined on $\mathcal{R}_{n, 1}$ and $\mathcal{C}$ respectively. Hence we find an identity due to the conformal transformation, ${ }^{4}$

$$
\langle\mathcal{O}(z)\rangle_{\mathcal{R}_{n, 1}}=\left\langle\left(\omega^{\prime}\right)^{h_{\mathcal{O}}} \mathcal{O}(\omega)+\cdots\right\rangle_{\mathcal{C}}
$$

$\omega^{\prime}$ is the derivative to $z, h_{\mathcal{O}}$ is the conformal dimension of $\mathcal{O}, \cdots$ is the inhomogeneous term in the conformal transformation for a general quasi-primary operator. Let $N=1$ in (2.7) and compare it with (2.9), we can solve the three point correlator $\left\langle\mathcal{O} \sigma_{n} \tilde{\sigma}_{n}\right\rangle$. For example, for the stress tensor, the Schwarzian derivative will contribute to $\left\langle\mathcal{T} \sigma_{n} \tilde{\sigma}_{n}\right\rangle$ and the result is consistent with those from Ward identity of $\mathcal{T}$ with two primary operator with conformal dimension $h=\frac{c}{24}\left(n-\frac{1}{n}\right)$. This fact actually determines the partition function $Z_{n, 1}$, hence the one-interval Rényi entropy and entanglement entropy are

$$
S_{[u, v]}^{(n)}=\frac{c(n+1)}{6 n} \log \frac{|u-v|}{\epsilon}, \quad S_{[u, v]}=\frac{c}{3} \log \frac{|u-v|}{\epsilon} .
$$

When the system is at a finite temperature $\frac{1}{\beta}$, there is another conformal transformation which maps the complex plane to the cylinder,

$$
s=\frac{\beta}{2 \pi} \log z
$$

\footnotetext{
${ }^{3}$ We add a subscript $N$ to denote the number of intervals. Correspondingly, the $n$-sheeted Riemann surface is now denoted as $\mathcal{R}_{n, N}$.

${ }^{4}$ We just label the holomorphic part.
} 
where $s$ is a cylinder coordinate with $^{5} s=\sigma+i \tau, \sigma \in(-\infty, \infty), \tau \in[0, \beta)$. Then the transformation of the twist operator under (2.11) leads to the one-interval Rényi entropy and entanglement entropy at finite temperature,

$$
S_{\beta}^{(n)}([u, v])=\frac{c(n+1)}{6 n} \log \frac{\beta}{\pi \epsilon} \sinh \frac{\pi|u-v|}{\beta}, \quad S_{\beta}([u, v])=\frac{c}{3} \log \frac{\beta}{\pi \epsilon} \sinh \frac{\pi|u-v|}{\beta} .
$$

For one-interval, the essential part is the existence of a conformal map (2.8). This conformal map is multi-valued, whose $j$-th solution is

$$
\omega_{j}(z)=\omega_{0}(z) \exp ^{\frac{2 \pi i j}{n}}, j=0,1, \cdots, n-1,
$$

where $\omega_{0}(z)=\left(\frac{z-u}{z-v}\right)^{\frac{1}{n}}$.

Before we close this section, we emphasize that the conclusion (2.10) and (2.12) rely on the partition function is ${ }^{6}$

$$
Z_{1}=t r q^{L_{0}-\frac{c}{24}}
$$

where $q=e^{2 \pi i \tau}$. Once there is another continuous global symmetry, for instance, a higher spin symmetry, the partition function (2.14) should be deformed to

$$
Z_{1}=\operatorname{trq}^{L_{0}-\frac{c}{24}} e^{-\mu \int W} .
$$

One can expand the modified partition function according to the order of the chemical potential, and solve the problem perturbatively. There is another version of the modified partition function in the literature [38, 39], which is defined by inserting a zero mode of the higher spin current to $(2.14)$

$$
Z_{1}=\operatorname{trq}^{L_{0}-\frac{c}{24}} e^{2 \pi i \alpha W_{0}},
$$

where the parameter $\alpha=\mu \bar{\tau}$. However, the two ways of introducing the higher spin chemical potentials in (2.15) and (2.16) are not manifestly equivalent ${ }^{7}$ [40]. (2.15) and (2.16) may corresponds to canonical and holomorphic partition functions respectively. In this work, we will use the deformation defined by (2.15) for our computation. Hence, one should be careful to compare our CFT result with the holomorphic result in the gravity side. However, we have checked that (2.15) and (2.16) are actually the same up to order $\mu^{4}$, including the quantum corrections, please find more details in appendix D. Hence, at least up to order $\mu^{4}$, we can trust the classical and quantum results in this work. However, it is still an open issue to explain why we can obtain the same answer.

\section{$3 \quad \mathrm{AdS}_{3}$ higher spin gravity}

The $\mathrm{AdS}_{3}$ nonlinear higher spin gravity [12] describes the interaction between scalars(and fermions when promoting supersymmetry) and an infinite tower of spins with $s \geq 1$. The

\footnotetext{
${ }^{5}$ Here $\sigma$ is the spatial coordinate in the cylinder. One should distinguish it from the twist operator $\sigma_{n}$.

${ }^{6}$ There is always a barred sector here and below.

${ }^{7}$ We thank the anonymous referee for these helpful comments.
} 
matter multiplet can be truncated and one finds pure higher spin $\mathrm{AdS}_{3}$ theory. This pure higher spin theory is described by two flat connection equations

$$
F=\bar{F}=0,
$$

where $^{8} F=d A+A^{2}$ and the connection one form $A$ is valued in some higher spin algebra, which includes spin 2 algebra $s l(2)$ as a subalgebra. A well-known higher spin algebra is $h s[\lambda]$ which includes each spin $s \geq 2$ once. Another view is to write $3 \mathrm{~d}$ gravity as a difference of two Chern-Simons theory [17, 18],

$$
S_{g r}=S_{C S}[A]-S_{C S}[\bar{A}],
$$

with the Chern-Simons action $S_{C S}[A]=\frac{k_{c s}}{4 \pi} \int \operatorname{Tr}\left(A d A+\frac{1}{3} A^{3}\right), A$ is valued in $s l(2) . k_{c s}$ is related to Newton constant by comparing (3.2) to Einstein-Hilbert action. The pure higher spin gravity is constructed by embedding $s l(2)$ into a larger algebra [19].

\subsection{Asymptotic symmetry and $\mathcal{W}$ algebra}

By gauge fixing and imposing the extended asymptotic $\mathrm{AdS}_{3}$ boundary condition, one finds that the asymmetric symmetry is generated by a classical $\mathcal{W}$ algebra [20, 21]. Hence the $\mathrm{CFT}_{2}$ dual theory have a $\mathcal{W}$ symmetry. A $\mathcal{W}$ algebra can be understood as adding some higher spin primary fields to Virasoro algebra. The quantum version of the classical $\mathcal{W}$ algebra can be found by imposing Jacobi identity condition. For the purpose of our discussion, we only give the first few OPEs of quantum $\mathcal{W}_{\infty}(\lambda)$ as follows,

$$
\begin{aligned}
\mathcal{T}(z) \mathcal{T}(0) & \sim \frac{c / 2}{z^{4}}+\frac{2 \mathcal{T}}{z^{2}}+\frac{\partial \mathcal{T}}{z} \\
\mathcal{T}(z) \mathcal{W}(0) & \sim \frac{3 \mathcal{W}}{z^{2}}+\frac{\partial \mathcal{W}}{z} \\
\mathcal{T}(z) \mathcal{U}(0) & \sim \frac{4 \mathcal{U}}{z^{2}}+\frac{\partial \mathcal{U}}{z} \\
\frac{1}{\mathcal{N}_{3}} \mathcal{W}(z) \mathcal{W}(0) & \sim \frac{1}{z^{6}}+\frac{\frac{6}{c} \mathcal{T}}{z^{4}}+\frac{\frac{3}{c} \partial \mathcal{T}}{z^{3}}+\frac{\frac{12}{c} \mathcal{U}+\frac{9}{10 c} \partial^{2} \mathcal{T}+\frac{96}{c(5 c+22)} \Lambda}{z^{2}} \\
& +\frac{\frac{6}{c} \partial \mathcal{U}+\frac{1}{5 c} \partial^{3} \mathcal{T}+\frac{48}{c(5 c+22)} \partial \Lambda}{z} \\
\mathcal{W}(z) \mathcal{U}(0) & \sim \frac{12\left(\lambda^{2}-9\right)}{5\left(\lambda^{2}-4\right)}\left(\frac{\mathcal{W}}{z^{4}}+\frac{1}{3} \frac{\partial \mathcal{W}}{z^{3}}+\frac{1}{14} \frac{\partial^{2} \mathcal{W}}{z^{2}}+\frac{1}{84} \frac{\partial^{3} \mathcal{W}}{z}+\cdots\right)+\cdots \\
\mathcal{U}(z) \mathcal{U}(0) & \sim \frac{c\left(\lambda^{2}-9\right)}{5\left(\lambda^{2}-4\right)} \frac{1}{z^{8}}+\cdots
\end{aligned}
$$

Where $\mathcal{T}, \mathcal{W}$ and $\mathcal{U}$ are spin 2 , spin 3 and spin 4 operators correspondingly. $\Lambda$ is a composite quasi-primary operator which is defined as $\Lambda=: \mathcal{T} \mathcal{T}:-\frac{3}{10} \partial^{2} \mathcal{T}$. In the last two OPEs, we just list the terms which are relevant to our discussions below. The normalization constant $\mathcal{N}_{3}$ can be chosen freely. When $\mathcal{N}_{3}=c / 3$, These quantum $\mathcal{W}_{\infty}(\lambda)$ algebra is the same

\footnotetext{
${ }^{8}$ In this paper, we always omit the bar term to simplify notation.
} 
as [22]. In the following discussion, we will choose $\mathcal{N}_{3}=-\frac{5 c}{6 \pi^{2}}$. There is a free parameter $\lambda$, which is related to the higher spin algebra $h s[\lambda]$ in the bulk.

In a conformal field theory, for a primary operator $\mathcal{O}_{i}$ with dimension $h_{i}$, the two, three and four point functions are respectively

$$
\begin{aligned}
\left\langle\mathcal{O}_{i}\left(z_{1}\right) \mathcal{O}_{j}\left(z_{2}\right)\right\rangle_{\mathcal{C}} & =\delta_{i j} \frac{\mathcal{N}_{\mathcal{O}_{i}}}{z_{12}^{2 h}}, \\
\left\langle\mathcal{O}_{i}\left(z_{1}\right) \mathcal{O}_{j}\left(z_{2}\right) \mathcal{O}_{k}\left(z_{3}\right)\right\rangle_{\mathcal{C}} & =\frac{C_{\mathcal{O}_{i} \mathcal{O}_{j} \mathcal{O}_{k}}}{z_{12}^{h_{i}+h_{j}-h_{k}} z_{23}^{h_{j}+h_{k}-h_{i}} z_{31}^{h_{k}+h_{i}-h_{j}}}, \\
\left\langle\mathcal{O}_{i}\left(z_{1}\right) \mathcal{O}_{j}\left(z_{2}\right) \mathcal{O}_{k}\left(z_{3}\right) \mathcal{O}_{l}\left(z_{4}\right)\right\rangle_{\mathcal{C}} & =\frac{\left(\frac{z_{24}}{z_{14}}\right)^{h_{i}-h_{j}}\left(\frac{z_{13}}{z_{14}}\right)^{h_{k}-h_{l}}}{z_{12}^{h_{i}+h_{j}} z_{34}^{h_{k}+h_{l}}} f_{i j k l}(x),
\end{aligned}
$$

with $z_{i j}=z_{i}-z_{j}$. They are determined by global conformal symmetry. The constants $\mathcal{N}_{\mathcal{O}_{i}}, C_{\mathcal{O}_{i} \mathcal{O}_{j} \mathcal{O}_{k}}$ can be read from the $\mathcal{O}_{i} \mathcal{O}_{j}$ OPE. And $f_{i j k l}$ is a function of the cross ratio $x=\frac{z_{13} z_{24}}{z_{14} z_{23}}$. For the theory with $\mathcal{W}_{\infty}(\lambda)$ symmetry, we find

$$
\mathcal{N}_{\mathcal{W}}=\mathcal{N}_{3}, \quad C_{\mathcal{W W W}}=0
$$

and

$$
f_{\mathcal{W W W} \mathcal{W}}=\mathcal{N}_{3}^{2} \sum_{i=0}^{6} a[3, j] \theta^{j},
$$

where $\theta\left(z_{1}, \cdots, z_{4}\right)=x+\frac{1}{x}-2=\frac{z_{12}^{2} z_{34}^{2}}{z_{13} z_{14} z_{23} z_{24}}$. The constants $a[3, j]$ are respectively

$$
\begin{aligned}
& a[3,0]=1, \quad a[3,1]=\frac{18}{c}, \quad a[3,2]=-\frac{9(c-98)}{c(5 c+22)}+\frac{144\left(\lambda^{2}-9\right)}{\left(\lambda^{2}-4\right) 5 c}, \\
& a[3,3]=2+\frac{54}{c}, \quad a[3,4]=9+\frac{18}{c}, \quad a[3,5]=6, \quad a[3,6]=1 .
\end{aligned}
$$

Actually, the four point correlator of spin $J$ is

$$
\frac{\mathcal{N}_{J}^{2}}{z_{12}^{2 J} z_{34}^{2 J}} \sum_{j=0}^{2 J} a[J, j] \theta^{j} .
$$

This can be understood as follows. The exchange symmetry of 1 and 2 restrict the function $f(x)$ as a function of $\theta=\left(x+\frac{1}{x}\right)-2$, while the maximal power of $\theta$ is determined by examining the most singular behavior as $z_{1} \rightarrow z_{3}$. Constants $a[J, j]$ are found by matching $(3.15)$ with the spin $J$ four point function from the Ward identity.

\subsection{Higher spin black hole}

A higher spin black hole [23] is a solution of (3.1) which has higher spin charges and higher spin chemical potentials. Choosing the three coordinates to be $x^{ \pm}, \rho$, a spin 3 black hole in $\operatorname{sl}(3)$ theory is

$$
A=b^{-1}(a+d) b
$$


with

$$
\begin{aligned}
a & =a_{+} d x^{+}+a_{-} d x^{-}, & b & =e^{\rho L_{0}}, \\
a_{+} & =L_{1}-\frac{2 \pi}{k} \mathcal{L} L_{-1}-\frac{\pi}{2 k} \mathcal{W} W_{-2}, & a_{-} & \sim \mu\left(a_{+}^{2}-\frac{1}{3} \operatorname{tr} a_{+}^{2}\right) .
\end{aligned}
$$

Here we split the $\operatorname{sl}(3)$ generators into the spin $2 \operatorname{part}\left(L_{i}, i=0, \pm 1\right)$ and the spin 3 $\operatorname{part}\left(W_{m}, m=0, \pm 1, \pm 2\right)$. Two parameters $\mathcal{L}, \mathcal{W}$ are related to the spin 2 and spin 3 charges. ${ }^{9}$ The parameter $\mu$ is the spin 3 chemical potential. There is another implicit parameter in this solution, which is the inverse temperature $\tau$. It appears in the periodic identification ${ }^{10}$

$$
z \sim z+2 \pi, z \sim z+2 \pi \tau .
$$

To ensure the smoothness of the solution, one should impose trivial holonomy condition around the thermal circle. This condition relates the chemical potentials to the higher spin charges, hence there are two freely parameters in the smooth solutions. A spin 3 black hole in $h s[\lambda]$ theory can be constructed analogously.

Though the usual notion of geometry is lack in this context, one can still define consistent thermodynamics for higher spin black holes. So far, several methods have been developed to study the first law thermodynamics of higher spin black holes. These includes,

1. Dimensional analysis [26]. In this method, one requires the consistency of first law of thermodynamics and relies on the dimensional counting of the quantities in the theory. The striking formula follows from Euler's theorem on homogeneous functions. One can regard this method as a higher spin generalization of Smarr's formula [27] for usual black holes.

2. Action variational principle [28-30]. In this method, the action is thought as a saddle point approximation of the partition function. One adds suitable boundary terms to the action to ensure the variation of the action to be consistent with the first law of thermodynamics. It is a natural generalization of Gibbons-Hawking's analysis of black hole thermodynamics [31].

3. Conical singularity method [35]. The gravitational entropy can be calculated by the conical singularity [36, 37] method, the authors in [35] found an extension to the higher spin gravity.

4. Wilson line approach $[32,47]$. This method can be generalized to calculate classical holographic entanglement entropy straightforwardly, we will discuss it in more detail in the following sections.

5. Noether charge method [33]. After suitability reformulating the Noether charge method [34] in Chern-Simons language, the authors in [33] can define the entropy

\footnotetext{
${ }^{9}$ We should mention that in different formalism, the identification of the charges is different. But in any case they can be determined by these two parameters.

${ }^{10}$ Here we have transfered the solution to Euclidean version and replaced $x^{+}\left(x^{-}\right)$with $z(\bar{z})$.
} 
of higher spin black holes. It is interesting that another choice of the Killing parameter leads to a new entropy for a higher spin black hole. In this paper we will not discuss this case.

A higher spin black hole corresponds to a CFT ensemble at finite temperature and with (higher spin) chemical potentials. For a higher spin black hole with a spin 3 chemical potential turning on, assuming the chemical potential $\mu$ is small, ${ }^{11}$ one can evaluate the partition function and reproduce the tree level results in the gravity $\operatorname{side}^{12}[38,39]$. Here we give the perturbative partition function for spin 3 black hole in $h s[\lambda]$ theory,

$$
\log Z=\frac{i \pi c}{12 \tau}\left(1-\frac{4}{3}\left(\frac{\alpha}{\tau^{2}}\right)^{2}+\frac{400\left(\lambda^{2}-7\right)}{27\left(\lambda^{2}-4\right)}\left(\frac{\alpha}{\tau^{2}}\right)^{4}+\cdots\right)+\text { quantum correction. }
$$

Here $\alpha=-\mu \tau$. For $\lambda=3$, it reproduces the partition function of $\operatorname{sl}(3)$ black hole. The order $\mathcal{O}(c)$ part can be viewed as the tree level result and can be found either from gravity side $[38]^{13}$ or CFT side $[38,39]$. The quantum correction begins with $\mathcal{O}\left(c^{0}\right)$, it should be there and there is no explicit result so far. In our perturbative approach, we will find quantum corrections at the order $\mu^{4}$. The partition function should be [44]

$$
Z=\left\langle\exp \left(-\mu \int \mathcal{W}\right)\right\rangle_{\beta}
$$

We have used a subscript $\beta$ to represent the thermal ensemble. One can expand the exponential according to the order of $\mu$ and calculate the partition function directly,

$$
\log Z=\log Z_{0}+\frac{\mu^{2}}{2} \iint\langle\mathcal{W W}\rangle_{\beta}+\cdots
$$

$Z_{0}$ is the thermal partition function without higher spin deformation. We will develop this perturbation method to the order $\mu^{4}$ and find the exact answer in the following section. Here "exact" means that we can calculate the all loop higher spin thermodynamics at a finite order $\mu^{k}$, including the tree level and quantum corrections to the partition function. The tree level results (proportional to central charge $c$ ) will reproduce the gravity answer.

\section{Holographic HSEE}

As discussed in section 2, we can define the entanglement entropy for a QFT. Due to AdS/CFT correspondence, there should be a concept which is dual to entanglement entropy in CFT. In [46], the authors introduced holographic entanglement entropy(HEE) to resolve this problem. In short, for Einstein-Hilbert theory, the static HEE is the minimal area of the surfaces which is homologous to the boundary region A, up to a coefficient $\frac{1}{4 G_{N}}$. In $A d S_{3} / C F T_{2}$, for one-interval $[u, v]$, this is just the length of the geodesics which

\footnotetext{
${ }^{11}$ More precisely, the dimensionless quantity $\frac{\mu}{\tau} \ll 1$.

${ }^{12}$ Some works on higher spin partition function can also be found in [41, 42].

${ }^{13}$ In the gravity side, there are canonical formalism or holomorphic formalism, the result we list above is from holomorphic formalism. But one expects the same answer after one identifies the charges and potentials properly in the canonical formalism [43].
} 
connects the points $u$ and $v$. But in this case the story is more interesting. The entanglement entropy is also equal to the logarithmic of a Wilson line [32, 47] which connects the two points $u$ and $v$. Since Wilson line can be defined for arbitrary higher spin theory, it is natural to conjecture this object is the holographic HSEE. We discuss [47] in detail, since it also provides the holomorphic result.

The one-interval holographic HSEE is conjectured to be

$$
S_{A}=\left.\frac{k_{c s}}{\sigma_{1 / 2}} \log \lim _{\rho_{0} \rightarrow \infty} W_{R}(P, Q)\right|_{\rho_{P}=\rho_{Q}=\rho_{0}},
$$

where $P$ and $Q$ are two bulk points. When $\rho_{0} \rightarrow \infty$, they tend to the points $u$ and $v \cdot \sigma_{1 / 2}$ is a constant and can be determined by the theory. The Wilson line $W_{R}(P, Q)$ is defined to be

$$
W_{R}(P, Q)=\operatorname{tr}_{R}\left[\mathcal{P} \exp \int_{Q}^{P} \bar{A} \mathcal{P} \exp \int_{P}^{Q} A\right],
$$

where $\mathcal{P}$ means path ordering and $R$ denotes the representation. The representation can be found by matching the HEE to thermal entropy. In the holomorphic formalism, Wilson line (4.2) is replaced by

$$
W_{R}(P, Q)=\operatorname{tr}_{R}\left[\mathcal{P} \exp \int_{Q}^{P} \bar{A}_{-} d x^{-} \mathcal{P} \exp \int_{P}^{Q} A_{+} d x^{+}\right] .
$$

For spin 3 black hole in $s l(3)$ theory, $\sigma_{1 / 2}=1$ and $R$ is chosen to be the adjoint representation. The HSEE in the holomorphic formalism is

$$
\begin{aligned}
S(\Delta)= & \frac{1}{3} c \log \left[\frac{\beta}{\pi \epsilon} \sinh \frac{\pi \Delta}{\beta}\right]+\frac{4 c \pi^{2} \mu^{2}}{9 \beta^{2}(-1+U)^{4}} \sum_{i=0}^{2} e[2, i] \log ^{i}[U] \\
& +\frac{32 c \mu^{4} \pi^{4}}{27 \beta^{4}(-1+U)^{8}} \sum_{i=0}^{4} e[4, i] \log ^{i}[U]+\mathcal{O}\left(\mu^{6}\right),
\end{aligned}
$$

where $U=e^{\frac{2 \pi \Delta}{\beta}}$ and $\Delta=|u-v|$. We have expanded the HSEE to order $\mu^{4}$. The first term on the right hand side is the usual entanglement entropy (2.12). There is no contribution from odd power of $\mu$. Actually this is related to the fact that the correlation function of odd number of spin 3 operators is zero in the CFT side. For each even power of $\mu$, the $\left(\frac{\mu}{\beta}\right)^{2 k}$ correction is of the form

$$
\sum_{i=0}^{2 k} g[2 k, i ; U] \log ^{i}[U]
$$

where $g[2 k, i ; U]$ is a rational function of $\mathrm{U}$. In the limit $U \rightarrow \infty$, the terms $g[2 k, 1 ; U] \log [U]$ dominate. This is because that the HSEE should be proportional to the thermal entropy in the large interval limit,

$$
S(\Delta) \rightarrow s_{\text {thermal }} \Delta, \text { as } \Delta \rightarrow \infty
$$


We have rewritten $g[2, i ; U], g[4, i ; U]$ in (4.4) in terms of the functions $e[2, i ; U]$ and $e[4, i ; U]$. They are defined to be

$$
\begin{aligned}
& e[2,0 ; U]=-(-1+U)^{2}\left(5+2 U+5 U^{2}\right), \\
& e[2,1 ; U]=4\left(-1-U+U^{3}+U^{4}\right), \\
& e[2,2 ; U]=-6\left(U+U^{3}\right), \\
& e[4,0 ; U]=-(-1+U)^{4}\left(43-52 U+162 U^{2}-52 U^{3}+43 U^{4}\right), \\
& e[4,1 ; U]=8(-1+U)^{3}\left(5+12 U+19 U^{2}+19 U^{3}+12 U^{4}+5 U^{5}\right), \\
& e[4,2 ; U]=-4(-1+U)^{2} U\left(43+26 U+78 U^{2}+26 U^{3}+43 U^{4}\right), \\
& e[4,3 ; U]=12 U\left(-3-8 U+U^{2}-U^{4}+8 U^{5}+3 U^{6}\right), \\
& e[4,4 ; U]=-3 U\left(1+8 U+7 U^{2}+16 U^{3}+7 U^{4}+8 U^{5}+U^{6}\right) .
\end{aligned}
$$

Before ending this section, we remark that the Wilson line conjecture only reproduce the $\mathcal{O}(c)$ part of the HSEE. It would be an interesting issue to find out the quantum correction in the bulk side.

\section{Partition function of higher spin black hole}

The first step towards the HSEE is to find the higher spin partition function as indicated by formula (2.3). We develop a different method to calculate (3.20), including its quantum correction. This is also a warmup exercise before we tackle the more tough problem on HSRE and HSEE.

Let us consider a spin $J$ chemical potential in (3.21). We expand the exponential in (3.21), using the fact (3.9)-(3.11) and (3.15) on the correlator of spin $J$ operator and the conformal map (2.11), then the partition function $\log Z$ can be written out order by order

$$
\begin{aligned}
& \mathcal{O}\left(\mu^{1}\right): 0 \\
& \mathcal{O}\left(\mu^{2}\right): \frac{\mu_{J}^{2}}{2} \mathcal{N}_{J}\left(\frac{2 \pi}{\beta}\right)^{2 J}\left(\frac{\beta^{2}}{2 \pi}\right)^{2} I_{2}[J] \\
& \mathcal{O}\left(\mu^{3}\right): \frac{\mu_{J}^{3}}{6} C_{J J J}\left(\frac{2 \pi}{\beta}\right)^{3 J}\left(\frac{\beta^{2}}{2 \pi}\right)^{3} I_{3}[J] \\
& \mathcal{O}\left(\mu^{4}\right): \frac{\mu_{J}^{4}}{24} \mathcal{N}_{J}^{2}\left(\frac{2 \pi}{\beta}\right)^{4 J}\left(\frac{\beta^{2}}{2 \pi}\right)^{4} \sum_{j=0}^{2 J} a[J, j] I_{4}[J, j]-\frac{\mu^{4}}{8} \mathcal{N}_{J}^{2}\left(\frac{2 \pi}{\beta}\right)^{4 J}\left(\frac{\beta}{2 \pi}\right)^{4}\left(I_{2}[J]\right)^{2}
\end{aligned}
$$

where $\mathcal{N}_{J}, C_{J J J}, a[J, j]$ are constants which are determined by two, three and four spin $J$ correlation functions. Please find the details on $\mathcal{N}_{J}, C_{J J J}, a[J, j]$ in subsection 3.1. The integrals $I_{2}[J], I_{3}[J], I_{4}[J, j]$ are defined respectively as

$$
\begin{aligned}
I_{2}[J] & =\int d t_{1} d t_{2} \frac{\left(t_{1} t_{2}\right)^{J-1}}{t_{12}^{2 J}}, \\
I_{3}[J] & =\int d t_{1} d t_{2} d t_{3} \frac{\left(t_{1} t_{2} t_{3}\right)^{J-1}}{\left(t_{12} t_{23} t_{31}\right)^{J}}, \\
I_{4}[J, j] & =\int d t_{1} d t_{2} d t_{3} d t_{4} \frac{\left(t_{1} t_{2} t_{3} t_{4}\right)^{J-1}}{t_{12}^{2 J-2 j} t_{34}^{2 J-2 j} t_{13}^{j} t_{14}^{j} t_{23}^{j} t_{24}^{j} .}
\end{aligned}
$$


All the integrals are definite integrals ranging from 0 to $\infty$. Let us explain (5.2)-(5.7) in more detail. The integral in (3.21) is over the whole cylinder

$$
\int W=\int_{0}^{\beta} d \tau \int_{-\infty}^{\infty} d \sigma W
$$

The integral of $W$ in the spatial direction is just a conserved charge, hence we first anticipate the integral of $\tau$ to yield a factor $\beta$. To integrate $\sigma$, we use the coordinate transformation $t=e^{\frac{2 \pi}{\beta} \sigma}$, hence the integral replacement rule is

$$
\int_{0}^{\beta} d \tau \int_{-\infty}^{\infty} d \sigma=\frac{\beta^{2}}{2 \pi} \int_{0}^{\infty} \frac{d t}{t}
$$

In perturbation expansion (5.2)-(5.4), the factor $\frac{2 \pi}{\beta}$ is from the conformal map (2.11), whilst the factor $\frac{\beta^{2}}{2 \pi}$ is from (5.9). The two dimensional integrations in $I_{2}$ and $I_{4}$ become one dimensional integral ranging from 0 to $\infty$.

However, there are divergences originating from the infinite length of the cylinder. We introduce an IR cutoff by setting the length of the cylinder to be L. Define $M=\frac{2 \pi L}{\beta}$, then the answer can be written as a function of $\mathrm{M}$.

For spin 3 , the three point function is zero. We need to evaluate $I_{2}[3], I_{4}[3, j](j=$ $0, \cdots, 6)$, they are

$$
\begin{aligned}
& I_{2}[3]=-\frac{M}{30}, \quad I_{4}[3,0]=\frac{M^{2}}{900}, \\
& I_{4}[3,2]=\frac{M}{36}, \quad I_{4}[3,3]=-\frac{M}{36}+\frac{M^{3}}{630}, \\
& I_{4}[3,5]=-\frac{3 M}{8}+\frac{2 M^{3}}{105}, \quad I_{4}[3,6]=\frac{49 M}{36}-\frac{22 M^{3}}{315}+\frac{M^{2}}{450} . \\
& \begin{array}{l}
I_{4}[3,1]=\frac{7 M}{648}+\frac{M^{3}}{1890} \\
I_{4}[3,4]=\frac{17 M}{162}-\frac{M^{3}}{189}
\end{array}
\end{aligned}
$$

Including the spin 3 coefficients (3.14), we find the partition function to be

$$
\frac{2 \pi}{L} \ln Z_{\mathrm{BH}}=\frac{i \pi c}{12 \tau}\left[1-\frac{4}{3}\left(\frac{\alpha}{\tau^{2}}\right)^{2}+\frac{80\left(-1154+206 \lambda^{2}+25 c\left(-7+\lambda^{2}\right)\right.}{27(22+5 c)\left(-4+\lambda^{2}\right)}\left(\frac{\alpha}{\tau^{2}}\right)^{4}+\cdots\right] .
$$

The interesting fact is that the higher order divergence cancel, leaving out the linear divergence, which shows the extensive property of the partition function. If we choose the large c limit, $c \rightarrow \infty$, the $\mathcal{O}(c)$ partition function is exactly the same in (3.20). We can also read out the quantum correction at $\mathcal{O}\left(\mu^{4}\right),{ }^{14}$

$$
\left.\log Z\right|_{\mu^{4}, \text { quan }}=\frac{i 640 \pi c}{27(22+5 c) \tau}\left(\frac{\alpha}{\tau}\right)^{4}
$$

Some remarks follow.

1. At the order $\mu^{4}$, the quantum correction to the higher spin black hole partition function is independent of $\lambda$.

\footnotetext{
${ }^{14}$ This is the contribution of the holomorphic part. There should be a similar anti-homomorphic part.
} 
2. In the large $c$ limit, the quantum correction contributes a finite term

$$
\frac{128 i \pi}{27 \tau}\left(\frac{\alpha}{\tau}\right)^{4}
$$

This is the one-loop contribution. Of course, one can expand (5.11) in terms of $1 / c$ and find the higher loop contribution. (5.11) contains all loop corrections to the partition function of spin 3 black hole at $\mathcal{O}\left(\mu^{4}\right)$.

3. When we use another description (2.16), we find the same partition function of higher spin black hole up to $\mathcal{O}\left(\mu^{4}\right) .{ }^{15}$ This is included in appendix D.

\section{HSRE and HSEE}

In this section we compute the HSRE and HSEE to $\mathcal{O}\left(\mu^{4}\right)$. From (2.3), the unknown quantity is $\log Z_{n}$.

\section{1 $\mathcal{O}\left(\mu^{2}\right)$ correction}

As a first step, let us consider the $\mathcal{O}\left(\mu^{2}\right)$ correction. It is

$$
\frac{\mu_{J}^{2}}{2} \int d s_{1} d s_{2}\left\langle W_{J}\left(s_{1}\right) W_{J}\left(s_{2}\right)\right\rangle_{\mathcal{R}_{n, \beta}}=\frac{\mu_{J}^{2}}{2} \int d s_{1} d s_{2}\left(\frac{2 \pi}{\beta}\right)^{2 J} z_{1}^{J} z_{2}^{J}\left\langle W_{J}\left(z_{1}\right) W_{J}\left(z_{2}\right)\right\rangle_{\mathcal{R}_{n}}
$$

Here we use a spin $J$ chemical potential deformation. $\beta$ in the subscript means that we are in a thermal ensemble now. On the right hand side of the equality, we use the conformal map (2.11) from cylinder to complex plane. In this map, a point $s_{i}$ in the correlation function is mapped to ${ }^{16} z_{i}=e^{\frac{2 \pi s_{i}}{\beta}}$ and the end point of the interval $u, v$ are mapped to ${ }^{17}$ $l_{1}=e^{\frac{2 \pi u}{\beta}}, l_{2}=e^{\frac{2 \pi v}{\beta}}$. To find $\left\langle W_{J}\left(z_{1}\right) W_{J}\left(z_{2}\right)\right\rangle_{\mathcal{R}_{n}}$, we can use the conformal map (2.8) from $\mathcal{R}_{n}$ to complex plane. Since the theory is a $\mathbb{Z}_{n}$ orbifold theory, $W(z)=\sum_{j=0}^{n-1} W\left(z_{j}\right)$, the field in $j$-th sheet should be mapped to a value $\omega_{j}(z)$, hence

$$
\left\langle W_{J}\left(z_{1}\right) W_{J}\left(z_{2}\right)\right\rangle_{\mathcal{R}_{n}}=\sum_{j_{1}, j_{2}=0}^{n-1}\left(\frac{\partial \omega_{j_{1}}}{\partial z_{1}}\right)^{J}\left(\frac{\partial \omega_{j_{2}}}{\partial z_{2}}\right)^{J}\left\langle W_{J}\left(\omega_{j_{1}}\right) W_{J}\left(\omega_{j_{2}}\right)\right\rangle_{\mathcal{C}}
$$

The summation can be found by the residue theorem,

$$
\left\langle W_{J}\left(z_{1}\right) W_{J}\left(z_{2}\right)\right\rangle_{\mathcal{R}_{n}}=\frac{\mathcal{N}_{J}}{z_{12}^{2 J}} \sum_{j=0}^{J-1} b[J, j ; n] \xi^{j}
$$

\footnotetext{
${ }^{15}$ We thank the anonymous referee of this paper for suggesting such kind of check.

${ }^{16}$ Note the integral variables in the right hand side are still $s_{i}$, the coordinates $z_{i}$ should be understood as $z_{i}=z\left(s_{i}\right)=e^{\frac{2 \pi s_{i}}{\beta}}$.

${ }^{17}$ Since the Rényi and entanglement entropy is a function of the distance $|u-v|$, we can safely set $u=0, v=\Delta$. In this convention, the two points are mapped to $l_{1}=1, l_{2}=U$.
} 
where $\xi$ is defined to be $\xi=\frac{z_{12}^{2} l_{12}^{2}}{\left(z_{1}-l_{1}\right)\left(z_{2}-l_{1}\right)\left(z_{1}-l_{2}\right)\left(z_{2}-l_{2}\right)}$. Please find more details on this two point correlation function on $\mathcal{R}_{n}$ in appendix A. The $\mathcal{O}\left(\mu^{2}\right)$ correction to $\log Z_{n}$ is

$$
\left.\log Z_{n}\right|_{\mu^{2}}=\frac{\mu_{J}^{2}}{2} \mathcal{N}_{J}\left(\frac{2 \pi}{\beta}\right)^{2 J}\left(\frac{\beta^{2}}{2 \pi}\right)^{2} \sum_{j=0}^{J-1} b[J, j ; n](U-1)^{2 j} F[J, j],
$$

where the integral $F[J, j]$ is defined to be

$$
F[J, j]=\int d t_{1} d t_{2} \frac{\left(t_{1} t_{2}\right)^{J-1}}{t_{12}^{2 J-2 j}\left(t_{1}-1\right)^{j}\left(t_{1}-U\right)^{j}\left(t_{2}-1\right)^{j}\left(t_{2}-U\right)^{j}} .
$$

Due to the identities

$$
F[J, 0]=I_{2}[J], b[J, 0 ; n]=n,
$$

the $j=0$ term is cancelled in $n$-th Rényi entropy (2.3), so finally

$$
\left.S^{(n)}\right|_{\mu^{2}}=\frac{\mu_{J}^{2}}{2} \mathcal{N}_{J}\left(\frac{2 \pi}{\beta}\right)^{2 J}\left(\frac{\beta^{2}}{2 \pi}\right)^{2} \sum_{j=1}^{J-1} \tilde{b}[J, j ; n](U-1)^{2 j} F[J, j] .
$$

The constants $\tilde{b}[J, j ; n]=\frac{b[J, j ; n]}{1-n}$ whose value can be found in appendix A. The integral $F[J, j]$ can be evaluated, please find appendix B for details. There the reader can find the integral from spin 3 to spin 6 . For spin 3 , the $\mathcal{O}\left(\mu^{2}\right)$ correction of Rényi entropy is

$$
\begin{aligned}
\left.S_{\mathrm{spin} 3}^{(n)}\right|_{\mu^{2}}= & -\frac{8 \pi^{4} \mu^{2} \mathcal{N}_{3}}{\beta^{2}(U-1)^{4}} \times \\
& \times\left[\frac{1+n}{48 n}\left(c[3,1,0]+c[3,1,1] \log [U]+c[3,1,2] \log ^{2}[U]\right)\right. \\
& \left.\quad+\frac{-4-4 n+n^{2}+n^{3}}{240 n^{3}}\left(c[3,2,0]+c[3,2,1] \log [U]+c[3,2,2] \log ^{2}[U]\right)\right]
\end{aligned}
$$

The definition of $c[J, j, i]$ can be found in appendix B. Taking the limit $n \rightarrow 1$, the entanglement entropy is

$$
\left.S_{\text {spin } 3}\right|_{\mu^{2}}=-\frac{4 \pi^{4} \mu^{2} \mathcal{N}_{3}}{15 \beta^{2}(U-1)^{4}} \sum_{i=0}^{2} e[2, i] \log ^{i}[U]
$$

where $e[2, i]$ is the same ones in (4.4). Once we choose normalization convention $\mathcal{N}_{3}=-\frac{5 c}{6 \pi^{2}}$ and take into account of the contribution from anti-holomorphic part, (6.9) is equal to order $\mu^{2}$ term in (4.4).

As the function $b[J, j ; n]$ can be determined by the residue theorem and $F[J, j]$ can always be integrated out, the $\mu_{J}^{2}$ correction from the spin $J$ deformation to the Rényi and entanglement entropy can be obtained. In appendix C, we list these entropies for other spins, including the spin 4,5 and 6 cases. 


\section{$6.2 \mathcal{O}\left(\mu^{3}\right)$ correction}

We just introduce the method briefly as the three point function of spin 3 field is vanishing. However, for other kinds of fields or when there are many chemical potentials, the three point function may not be zero. So they can contribute to the Rényi and entanglement entropy. There is only one single chemical potential $\mu_{J}$ in our example, but the reader can extend it to the cases with arbitrary number of chemical potentials.

As in the previous subsection, we first map finite temperature $n$-sheeted Riemann surface $\mathcal{R}_{n, \beta}$ to $\mathcal{R}_{n}$ and then map the $\mathcal{R}_{n}$ to complex plane. After carefully collecting the conformal transformation factor and noticing the multi-value of the second transformation, we find the correction is

$$
\begin{aligned}
\left.\log Z_{n}\right|_{\mu_{J}^{3}}=\frac{C_{J J J} \mu_{J}^{3}}{6}\left(\frac{\pi}{\beta}\right)^{3 J}\left(\frac{\beta^{2}}{2 \pi}\right)^{3} \times \\
\quad \times \int d t_{1} d t_{2} d t_{3} \frac{(U-1)^{3 J}}{n^{3 J}} f\left[J, t_{1}\right] f\left[J, t_{2}\right] f\left[J, t_{3}\right] S\left[J, J, J ; x_{12}, x_{23}, x_{31}\right]
\end{aligned}
$$

The function $f[J, t]$ is defined as

$$
f[J, t]=\frac{t^{J-1}}{(t-1)^{J}(t-U)^{J}}
$$

and $x_{i j}$ is

$$
x_{i j}=\frac{\left(t_{i}-1\right)\left(t_{j}-U\right)}{\left(t_{j}-U\right)\left(t_{j}-1\right)} .
$$

The summation function $S[a, b, c ; x, y, z]$ is more involved, please find it in appendix A. The $\mu^{3}$ correction depends only on the three point function of the operators. The general type of integration is

$$
\int d t R[t] \log [t]^{k}
$$

with $k \leq 2$ and $R[t]=\frac{P[t]}{Q[t]}$ is just a rational function of $t$ where $P[t]$ and $Q[t]$ are polynomials of $t$. All such kind of integrals can be reduced to those discussed in appendix B. For spin 3 , the three point function is just zero. At least for this special example, we need not do the summation and integral at all. But such kind of consideration is useful to compute the $\mu^{4}$ correction, which is indeed relevant even for spin 3.

\section{$6.3 \mathcal{O}\left(\mu^{4}\right)$ correction}

After some algebra, we find the partition function of $n$-sheeted Riemann surface $\mathcal{R}_{n}$ is

$$
\begin{aligned}
\left.\log Z_{n}\right|_{\mu_{J}^{4}}= & \frac{\mathcal{N}_{J}^{2} \mu_{J}^{4}}{24}\left(\frac{\pi}{n \beta}\right)^{4 J}\left(\frac{\beta^{2}}{2 \pi}\right)^{4}(U-1)^{4 J} \\
& \times\left\{\left(\prod_{i=1}^{4} \int d t_{i} f\left[J, t_{i}\right]\right) \times\right. \\
& \left.\quad \times \sum_{j=0}^{2 J} a[J, j] S\left[2 J-2 j, j, j, j, j, 2 J-2 j ; x_{12}, x_{13}, x_{14}, x_{23}, x_{24}, x_{34}\right]\right\} \\
& -\frac{1}{2}\left(\mathcal{O}\left(\mu_{J}^{2}\right)\right)^{2},
\end{aligned}
$$


where $\mathcal{O}\left(\mu_{J}^{2}\right)$ is (6.4). The definition of $S$ can be found in appendix A.3. The number of $\mathrm{S}$ increases linearly with spin $J$. We choose the smallest number, $J=3$ to study. In this case, there are 7 kinds of $S$. The number of independent terms is estimated of order $\mathcal{O}\left(10^{2}\right)$ for $j=1,2, \cdots, 6$ and $\mathcal{O}(10)$ for $j=0$. We note that the gravity result is of order c. While in Rényi entropy, there is $\mathcal{O}\left(c^{2}\right)$ contribution superficially. We expect this $\mathcal{O}\left(c^{2}\right)$ contribution to be vanish, especially when we compute the entanglement entropy. Note that one has to prove this fact from the CFT side in principal. However, we haven't proved it as the summation of the $j=6$ term is extremely difficult. Therefore we just throw out all the terms which is $\mathcal{O}\left(c^{2}\right)$ before computation. Next, we look for $\mathcal{O}(c)$ term, which is the combination ${ }^{18}$

$$
S[4,1,1,1,1,4]-\frac{1}{10} S[2,2,2,2,2,2]+3 S[0,3,3,3,3,0]+S[-2,4,4,4,4,-2]
$$

There are also quantum corrections, which are contributed from $j=2$ term.

\subsection{1 $\mathcal{O}(c)$}

As mentioned above, we need to evaluate $S$ for $j=1,2,3,4$. All of the terms in S can be written as the product of some functions like $\operatorname{coth}^{a_{i j}}\left(\log \sqrt{x_{i j}}\right)$ with $a_{i j}$ being positive integers, $1 \leq i<j \leq 4$. Using an identity

$$
\operatorname{coth}\left[\log \sqrt{x_{i j}}\right]=2 y_{i j}-1
$$

with

$$
y_{i j}=\frac{\left(t_{i}-1\right)\left(t_{j}-U\right)}{\left(t_{i}-t_{j}\right)(1-U)}
$$

then (6.15) becomes a function $\mathcal{P}\left(y_{i j}\right)$. One can find the function $\mathcal{P}\left(y_{i j}\right)$ in appendix A.3. Each term in $\mathcal{P}\left(y_{i j}\right)$ is

$$
y_{12}^{a} y_{i 3}^{b} y_{j 4}^{c}
$$

with $a, b, c$ are positive integers. Since $i<3, j<4$, there are actually six type of integrals in (6.14). Each type of integrals can be done, please find more details in appendix B. Finally, the answer is

$$
\begin{aligned}
\left.\log Z_{n}\right|_{\mu^{4}, c}=-\frac{3 \mathcal{N}_{3}^{2} \mu^{4} \pi^{8}}{64 c \beta^{4}} & \frac{1}{(U-1)^{8}} \frac{1}{n^{5}} \times \\
& \times\left(\sum_{j=0}^{3} \sum_{i=0}^{4} g_{j}[i ; U] n^{2 j} \log ^{i}[U]+\left(-\frac{16384 M}{135} n^{6}(U-1)^{8}\right)\right)
\end{aligned}
$$

\footnotetext{
${ }^{18}$ We choose $\mathcal{W}_{3}$ theory here, the $\mathcal{W}_{\infty}(\lambda)$ can be found later.
} 
with

$$
\begin{aligned}
& g_{3}[0 ; U]=\frac{2048}{675} \times\left(-12(-1+U)^{4}\left(1+128 U+630 U^{2}+128 U^{3}+U^{4}\right)\right) \\
& g_{3}[1 ; U]=\frac{2048}{675} \times 8(-1+U)^{3}\left(5+159 U+2500 U^{2}+2500 U^{3}+159 U^{4}+5 U^{5}\right) \\
& g_{3}[2 ; U]=-\frac{2048}{675} \times 48(-1+U)^{2} U\left(8+277 U+762 U^{2}+277 U^{3}+8 U^{4}\right) \\
& g_{3}[3 ; U]=\frac{2048}{675} \times 36 U\left(-1-84 U-421 U^{2}+421 U^{4}+84 U^{5}+U^{6}\right) \\
& g_{3}[4 ; U]=-\frac{2048}{675} \times U\left(1+224 U+2455 U^{2}+5296 U^{3}+2455 U^{4}+224 U^{5}+U^{6}\right) \\
& g_{2}[0 ; U]=-\frac{2048}{225} \times(-1+U)^{4}\left(5-2028 U-7234 U^{2}-2028 U^{3}+5 U^{4}\right) \\
& g_{2}[1 ; U]=-\frac{2048}{225} \times 24(-1+U)^{3} U\left(89+851 U+851 U^{2}+89 U^{3}\right) \\
& g_{2}[2 ; U]=\frac{2048}{225} \times 4(-1+U)^{2} U\left(173+3730 U+9114 U^{2}+3730 U^{3}+173 U^{4}\right) \\
& g_{2}[3 ; U]=-\frac{2048}{225} \times 4 U\left(-21-920 U-3737 U^{2}+3737 U^{4}+920 U^{5}+21 U^{6}\right) \\
& g_{2}[4 ; U]=\frac{2048}{225} \times U\left(3+296 U+2677 U^{2}+5328 U^{3}+2677 U^{4}+296 U^{5}+3 U^{6}\right) \\
& g_{1}[0 ; U]=-\frac{2048}{225} \times(-1+U)^{4}\left(21+2468 U+7070 U^{2}+2468 U^{3}+21 U^{4}\right) \\
& g_{1}[1 ; U]=\frac{2048}{225} \times 24(-1+U)^{3} U\left(129+875 U+875 U^{2}+129 U^{3}\right) \\
& g_{1}[2 ; U]=-\frac{2048}{225} \times 4(-1+U)^{2} U\left(293+4162 U+9162 U^{2}+4162 U^{3}+293 U^{4}\right) \\
& g_{1}[3 ; U]=\frac{2048}{225} \times 8 U\left(-21-554 U-1841 U^{2}+1841 U^{4}+554 U^{5}+21 U^{6}\right) \\
& g_{1}[4 ; U]=-\frac{2048}{225} \times 8 U\left(1+49 U+365 U^{2}+676 U^{3}+365 U^{4}+49 U^{5}+U^{6}\right) \\
& g_{0}[0 ; U]=\frac{4096}{675} \times 3(-1+U)^{4}\left(15+476 U+1178 U^{2}+476 U^{3}+15 U^{4}\right) \\
& g_{0}[1 ; U]=-\frac{4096}{675} \times 4(-1+U)^{3}\left(5+519 U+2716 U^{2}+2716 U^{3}+519 U^{4}+5 U^{5}\right) \\
& g_{0}[2 ; U]=\frac{4096}{675} \times 24(-1+U)^{2} U\left(38+385 U+774 U^{2}+385 U^{3}+38 U^{4}\right) \\
& g_{0}[3 ; U]=-\frac{4096}{675} \times 48 U\left(-3-55 U-151 U^{2}+151 U^{4}+55 U^{5}+3 U^{6}\right) \\
& \frac{4096}{675} \times 8 U\left(1+32 U+199 U^{2}+346 U^{3}+199 U^{4}+32 U^{5}+U^{6}\right) \\
& =
\end{aligned}
$$

There is a IR divergence term in $\left.\log Z_{n}\right|_{\mu^{4}, c}$. It is canceled by the similar term in $\left.n \log Z_{1}\right|_{\mu^{4}, c}$, so the Rényi entropy is finite at this order. We plug (6.19), (5.10) into ${ }^{19}(2.3)$ and find the $\mathcal{O}(c)$ Rényi entropy to be

$$
\left.S^{(n)}\right|_{\mu^{4}, c}=\frac{1}{n-1} \frac{3 \mathcal{N}_{3}^{2} \mu^{4} \pi^{8}}{64 c \beta^{4}} \frac{1}{(U-1)^{8}} \frac{1}{n^{5}} \times\left(\sum_{j=0}^{3} \sum_{i=0}^{4} g_{j}[i ; U] n^{2 j} \log ^{i}[U]\right) .
$$

\footnotetext{
${ }^{19}$ As we are dealing with CFT with $\mathcal{W}_{3}$ symmetry, we choose $\lambda=3$ here.
} 
Taking the limit $n \rightarrow 1$, the entanglement entropy is

$$
\left.S\right|_{\mu^{4}, c}=\frac{16 c \mu^{4} \pi^{4}}{27 \beta^{4}(-1+U)^{8}} \sum_{i=0}^{4} e[4, i] \log ^{i}[U] .
$$

Note that $e[4, i]$ are those in (4.4). After including the anti-holomorphic part, we reproduce exactly the large $c$ limit of HSEE (4.4)!

\subsubsection{Quantum correction}

As we have mentioned, there is quantum correction at $\mathcal{O}\left(\mu^{4}\right)$. It is from $j=2$ term in (6.14). So it is important to derive this term seperately. The method is the same as before. We give the result of $\left.\log Z_{n}\right|_{\mu^{4}, \text { quan }}$ below.

$$
\begin{aligned}
&\left.\log Z_{n}\right|_{\mu^{4}, \text { quan }}=-\frac{\left.\pi^{8} \mu^{4} \mathcal{N}_{3}^{2}(a[3,2])\right|_{\text {quan }}}{384 \beta^{4} n^{5}(U-1)^{8}} \times \\
& \times\left[\sum_{j=0}^{3} \sum_{i=0}^{4} \gamma_{j}[i ; U] n^{2 j} \log ^{i}[U]+\left(-\frac{1024 M}{9} n^{6}(U-1)^{8}\right)\right]
\end{aligned}
$$

where $\left.(a[3,2])\right|_{\text {quan }}$ is the quantum parts of $a[3,2]$,

$$
\left.(a[3,2])\right|_{\text {quan }}=\frac{4608}{5 c(22+5 c)} .
$$

That means the quantum correction to the Rényi and entanglement entropy at $\mathcal{O}\left(\mu^{4}\right)$ is universal in the sense that it is independent of $\lambda$. This is similar to the quantum correction to the thermal partition function, which is also $\lambda$ independent at this order. There is also a IR divergence term here, it is canceled by $\left.n \log Z_{1}\right|_{\mu^{4}, \text { quan }}$ exactly. So to find the Rényi entropy, we just delete the term related to $\mathrm{M}$ in (6.22) and then divide $1-n$. The functions $\gamma_{j}[i ; U]$ are

$$
\begin{aligned}
& \gamma_{3}[0 ; U]=\frac{256}{315} \times 4(-1+U)^{4}\left(7-704 U-4150 U^{2}-704 U^{3}+7 U^{4}\right) \\
& \gamma_{3}[1 ; U]=\frac{256}{315} \times 4(-1+U)^{3}\left(35+323 U+10730 U^{2}+10730 U^{3}+323 U^{4}+35 U^{5}\right) \\
& \gamma_{3}[2 ; U]=-\frac{256}{315} \times 12(-1+U)^{2} U\left(37+2128 U+6758 U^{2}+2128 U^{3}+37 U^{4}\right) \\
& \gamma_{3}[3 ; U]=\frac{256}{315} \times 4 U\left(-9-1316 U-8429 U^{2}+8429 U^{4}+1316 U^{5}+9 U^{6}\right) \\
& \gamma_{3}[4 ; U]=-\frac{256}{315} \times U\left(1+324 U+5095 U^{2}+11336 U^{3}+5095 U^{4}+324 U^{5}+U^{6}\right) \\
& \gamma_{2}[0 ; U]=-\frac{512}{45} \times 2(-1+U)^{4}\left(5-488 U-1914 U^{2}-488 U^{3}+5 U^{4}\right) \\
& \gamma_{2}[1 ; U]=-\frac{512}{45} \times 48(-1+U)^{3} U\left(19+221 U+221 U^{2}+19 U^{3}\right) \\
& \gamma_{2}[2 ; U]=\frac{512}{45} \times 24(-1+U)^{2} U\left(11+310 U+798 U^{2}+310 U^{3}+11 U^{4}\right) \\
& \gamma_{2}[3 ; U]=-\frac{512}{45} \times 4 U\left(-7-440 U-1979 U^{2}+1979 U^{4}+440 U^{5}+7 U^{6}\right)
\end{aligned}
$$




$$
\begin{aligned}
& \gamma_{2}[4 ; U]=\frac{512}{45} \times U\left(1+132 U+1359 U^{2}+2776 U^{3}+1359 U^{4}+132 U^{5}+U^{6}\right) \\
& \gamma_{1}[0 ; U]=-\frac{256}{45} \times(-1+U)^{4}\left(29+2932 U+8910 U^{2}+2932 U^{3}+29 U^{4}\right) \\
& \gamma_{1}[1 ; U]=\frac{256}{45} \times 24(-1+U)^{3} U\left(151+1085 U+1085 U^{2}+151 U^{3}\right) \\
& \gamma_{1}[2 ; U]=-\frac{256}{45} \times 12(-1+U)^{2} U\left(109+1706 U+3786 U^{2}+1706 U^{3}+109 U^{4}\right) \\
& \gamma_{1}[3 ; U]=\frac{256}{45} \times 4 U\left(-43-1352 U-4583 U^{2}+4583 U^{4}+1352 U^{5}+43 U^{6}\right) \\
& \gamma_{1}[4 ; U]=-\frac{256}{45} \times U\left(7+468 U+3585 U^{2}+6712 U^{3}+3585 U^{4}+468 U^{5}+7 U^{6}\right) \\
& \gamma_{0}[0 ; U]=\frac{256}{315} \times(-1+U)^{4}\left(315+9676 U+25378 U^{2}+9676 U^{3}+315 U^{4}\right) \\
& \gamma_{0}[1 ; U]=-\frac{256}{315} \times 4(-1+U)^{3}\left(35+3473 U+19172 U^{2}+19172 U^{3}+3473 U^{4}+35 U^{5}\right) \\
& \gamma_{0}[2 ; U]=\frac{256}{315} \times 24(-1+U)^{2} U\left(246+2695 U+5458 U^{2}+2695 U^{3}+246 U^{4}\right) \\
& \gamma_{0}[3 ; U]=-\frac{256}{315} \times 16 U\left(-53-1155 U-3201 U^{2}+3201 U^{4}+1155 U^{5}+53 U^{6}\right) \\
& \gamma_{0}[4 ; U]=\frac{256}{315} \times 4 U\left(9+438 U+2791 U^{2}+4864 U^{3}+2791 U^{4}+438 U^{5}+9 U^{6}\right)
\end{aligned}
$$

The quantum correction to the entanglement entropy is

$$
\left.S\right|_{\mu^{4}, \text { quan }}=\frac{512 c \mu^{4} \pi^{4}}{63 \beta^{4}(22+5 c)(-1+U)^{8}} \sum_{i=0}^{4} q[i ; U] \log ^{i}[U],
$$

where the functions $q[i ; U]$ are respectively

$$
\begin{aligned}
& q[0 ; U]=-(-1+U)^{4}\left(133+548 U+1662 U^{2}+548 U^{3}+133 U^{4}\right) \\
& q[1 ; U]=4(-1+U)^{3}\left(35+309 U+1168 U^{2}+1168 U^{3}+309 U^{4}+35 U^{5}\right) \\
& q[2 ; U]=-24\left((-1+U)^{2}\right) U\left(43+161 U+348 U^{2}+161 U^{3}+43 U^{4}\right) \\
& q[3 ; U]=16 U\left(-11-91 U-163 U^{2}+163 U^{4}+91 U^{5}+11 U^{6}\right) \\
& q[4 ; U]=-8 U\left(1+23 U+97 U^{2}+136 U^{3}+97 U^{4}+23 U^{5}+U^{6}\right)
\end{aligned}
$$

\subsection{3 $\mathcal{W}_{\infty}(\lambda)$ theory}

We find the difference between $\mathcal{W}_{\infty}(\lambda)$ and $\mathcal{W}_{3}$ theory is just the $j=2$ coefficient of $a[3, j]$ at $\mathcal{O}\left(\mu^{4}\right)$. So the Rényi and entanglement entropy of $\mathcal{W}_{\infty}(\lambda)$ theory can be read out directly without any further computation. The difference of the Rényi entropy between $\mathcal{W}_{\infty}(\lambda)$ and $\mathcal{W}_{3}$ theory is

$$
\left.S_{\mathcal{W}_{\infty}(\lambda)}^{(n)}-S_{\mathcal{W}_{3}}^{(n)}=\frac{1}{n-1} \frac{\pi^{8} \mu^{4} \mathcal{N}_{3}^{2} \delta}{384 \beta^{4} n^{5}(U-1)^{8}} \times\left[\sum_{j=0}^{3} \sum_{i=0}^{4} \gamma_{j}[i ; U] n^{2 j} \log ^{i}[U]\right)\right]+\mathcal{O}\left(\mu^{6}\right)
$$

Here $\delta$ is the difference of $a[3, j]$ for arbitrary $\lambda$ and $\lambda=3$, it is

$$
\delta=\frac{144\left(-9+\lambda^{2}\right)}{5 c\left(-4+\lambda^{2}\right)} .
$$


Taking the limit $n \rightarrow 1$ and choosing the normalization $\mathcal{N}_{3}$, we find the difference of the entanglement entropy is

$$
S_{\mathcal{W}_{\infty}(\lambda)}-S_{\mathcal{W}_{3}}=\frac{16 c \mu^{4} \pi^{4}\left(\lambda^{2}-9\right)}{63 \beta^{4}\left(\lambda^{2}-4\right)(-1+U)^{8}} \sum_{i=0}^{4} q[i ; U] \log ^{i}[U]+\mathcal{O}\left(\mu^{6}\right) .
$$

Note the difference is of $\mathcal{O}(c)$, indicating the quantum correction is the same for different $\lambda$ at this order. However, it is not clear whether this property holds to higher order of $\mu$.

\section{Conclusion and discussion}

In this work, we have developed a perturbation formulation to calculate HSRE and HSEE at finite temperature and with finite chemical potential. As suggested in [48], by using a multi-valued conformal map from $\mathcal{R}_{n}$ to complex plane, the correlation functions of primary operators on $\mathcal{R}_{n}$ is mapped to a multi-summation of the correlation functions of the same operators in the complex plane. After doing tedious summation and integration, we reproduced the universality property which is first observed in [44] and proved in [48] at the order $\mathcal{O}\left(\mu^{2}\right)$ up to a normalization constant. This university holds not only for spin 3 theory but also for arbitrary kinds of higher spin deformation and arbitrary number of higher spin deformations. The results for spin 4 to spin 6 are given in appendix C, but we can extend the computation to any spin without difficulty. We also check the holographic HSEE [32, 47] up to $\mathcal{O}\left(\mu^{4}\right)$ for $\mathcal{W}_{3}$ theory. This strongly supports the Wilson line prescription of HSEE.

Besides the confirmation of the existing results in the literature, there are many novel results from our study:

1. We can calculate not only HSEE but also HSRE. It would be interesting to develop the dual holographic computation of HSRE.

2. We obtained the quantum correction to HSRE and HSEE at order $\mathcal{O}\left(\mu^{4}\right)$ which is absent in the classical Wilson line prescription. We also find quantum correction to the partition function of higher spin black holes. These provides interesting results to probe the quantum property of higher spin gravity, for example, the fluctuation of fields on the higher spin black hole background.

3. For the higher spin black holes other than $s l(3)$ black hole, our method can also be used to calculate the HSRE and HSEE from CFT side and check the Wilson line prescription. However, as the rank of the gauge group increases, the holographic calculation becomes more difficult quickly. For the black holes appeared in $h s[\lambda]$ theory, the exist prescription $[32,47]$ faces technical problem due to the infinite dimension of the group. However, our CFT calculation overcome this difficulty without trouble. In fact, in this work, we have calculated the HSRE and HSEE for $\mathcal{W}_{\infty}(\lambda)$ up to $\mathcal{O}\left(\mu^{4}\right)$. In the holographic prescription, there is no explicit computation so far. We also found that at $\mathcal{O}\left(\mu^{4}\right)$, the difference of HSEE(and HSRE) between $\mathcal{W}_{\infty}$ and $\mathcal{W}_{3}$ theory is purely classical, without any quantum correction! It would be interesting to check this fact at higher order of $\mu$. 
4. There is another point we do not discuss extensively in this paper. When there are many higher spin potentials, the $\mathcal{O}\left(\mu^{3}\right)$ can be non-zero. Except for a theory dependent three point function constant $C_{J_{1} J_{2} J_{3}}$, our method indicates that the total correction of HSRE and HSEE at this order is also universal. This is similar to $\mu^{2}$ correction. In conformal field theory, the structure of two and three point function of primary operators are determined by global conformal symmetry, as shown in (3.9) and (3.10). This means the universal property found in $\mathcal{O}\left(\mu^{2}\right)$ and $\mathcal{O}\left(\mu^{3}\right)$ actually originates from the global conformal symmetry in the theory.

Besides the previous concrete conclusion, we can give some discussions below.

1. We can calculate the correction to arbitrary order $\mathcal{O}\left(\mu^{k}\right)$ with finite ${ }^{20} k>4$. To $\mathcal{O}\left(\mu^{k}\right)$, we only need to know the $k$-point function of higher spin operators which is determined by the Ward identity, this may be complicated but can be evaluated.

2. Our formulation does not use the usual prescription of the twist operator. Instead, we just used a conformal map from $n$-sheeted Riemann surface to complex plane. Since the twist operator method should give the same answer as here, one may read out some interesting information about the twist operator from our result. For instance, the most singular term of the OPE between a spin $J(J>2)$ operator $\mathcal{W}_{J}$ with twist operator $\sigma_{n}$ should be

$$
\mathcal{W}_{J}(z) \sigma_{n}(0) \sim \frac{\sigma_{n}^{\prime}(0)}{z^{J-1}}+\cdots
$$

where $\sigma_{n}^{\prime}$ is another primary operator with dimension $\frac{c}{24}\left(n-\frac{1}{n}\right)+1$. We expect to find a complete OPE between $\mathcal{W}_{J}$ and $\sigma_{n}$, with the results found in this work.

3. When the interval number $N>1$, there is no similar conformal map. In that case, we can not get the answer for all interval lengths. However, for short intervals,we can use all the technics [49-52] to calculate HSRE and HSEE.

4. Our method to evaluate HSEE(and HSRE) is perturbative, it give new results which is beyond the holographic method. However, we notice that the holographic description provides the classical correction to all order of $\mu$ in the large c limit. In CFT side, to get a result analogously is quite difficult due to the complicated summation and integrals shown in the appendices. It would be interesting to develop some new methods to evaluate the all order $\mu$ result from CFT side directly.

There are some open issues in the computation which are listed below.

1. In our computation, we throw out all the $\mathcal{O}\left(c^{2}\right)$ terms. Moreover, for $\mathcal{O}\left(\mu^{k}\right) k>4$ correction, there are many $\mathcal{O}\left(c^{l}\right)(l \geq 2)$ terms. It is still an open issue to prove that we can throw them out consistently.

2. When we compute the integrals, we use the replacement rules (B.6), (B.10), (B.12) by hand. This leads to the correct answer. A better understanding of these rules is valuable.

\footnotetext{
${ }^{20}$ Some technical subtleties will be discussed at the end.
} 


\section{Acknowledgments}

The author J.L thanks Zhuang-wei Jin, Feng-yan Song, Jie-qiang Wu and Jian-dong Zhang for their helpful supports during this work. J.L is especially thankful to prof. Bin Chen for helpful discussions and for his kind proof reading and comments on previous drafts. The work was in part supported by NSFC Grant No. 11275010.

\section{A Correlation function on $\mathcal{R}_{n}$}

\section{A.1 Two point function on $\mathcal{R}_{n}$}

In (6.3), we use the two point function in complex plane and notice the multi-valued solution of the conformal map (2.8), then

$$
\begin{aligned}
\left\langle W_{J}\left(z_{1}\right) W_{J}\left(z_{2}\right)\right\rangle_{\mathcal{R}_{n}}=\frac{\mathcal{N}_{J}}{(2 n)^{2 J}}\left(\frac{l_{12}^{2}}{\left(z_{1}-l_{1}\right)\left(z_{1}-l_{2}\right)\left(z_{2}-l_{1}\right)\left(z_{2}-l_{2}\right)}\right)^{J} \times \\
\quad \times \sum_{j_{1}, j_{2}=0}^{n-1} \frac{1}{\sinh ^{2 J}\left(\frac{\log \sqrt{x}}{n}+\frac{\pi i\left(j_{1}-j_{2}\right)}{n}\right)},
\end{aligned}
$$

where the cross ratio of the four points $z_{1}, z_{2}, l_{1}, l_{2}$ is defined to be

$$
x=\frac{\left(z_{1}-l_{1}\right)\left(z_{2}-l_{2}\right)}{\left(z_{1}-l_{2}\right)\left(z_{2}-l_{1}\right)} .
$$

(A.1)is universal for different theory up to a spin $J$ normalization. This confirms the conclusion in [48]. Let's define a function

$$
S[a ; x]=\sum_{j_{1}, j_{2}=0}^{n-1} \frac{1}{\sinh ^{2 a}\left(\frac{\log \sqrt{x}}{n}+\frac{\pi i\left(j_{1}-j_{2}\right)}{n}\right)} .
$$

Since the summation depends on the difference between $j_{1}$ and $j_{2}$, we get a factor $n$ by eliminating one summation index,

$$
S[a ; x]=n \sum_{j=0}^{n-1} \frac{1}{\sinh ^{2 a}\left(\frac{\log \sqrt{x}}{n}+\frac{\pi i j}{n}\right)} .
$$

Since $a$ is an integer, the summation can be converted to a contour integral,

$$
P[a ; x]=\oint_{\mathcal{C}} \frac{d z}{2 \pi i} \frac{1}{\sinh ^{2 a}\left(\frac{\log \sqrt{x}}{n}+z\right)} \operatorname{coth}(n z),
$$

in which the contour can be chosen as follows,

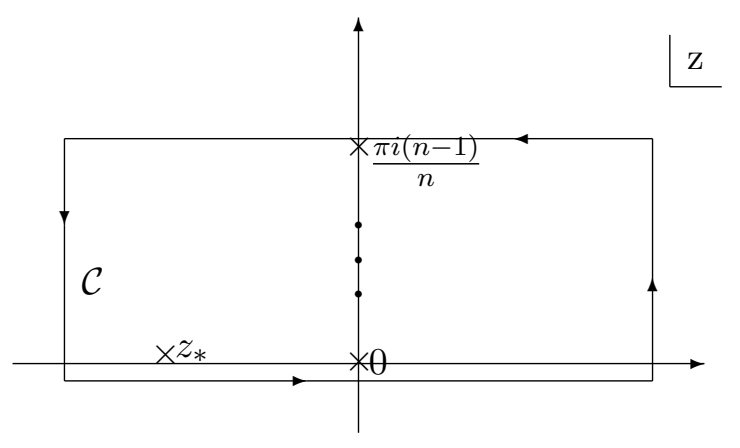


The contour $\mathcal{C}$ includes the singularities $z=\frac{\pi i j}{n}, j=0, \cdots, n-1$ and $z=z_{*}$, with $z_{*}=-\frac{\log \sqrt{x}}{n}$. The height of the contour is $\pi$, since under a shift of $z \rightarrow z+\pi i$ the function to be integrated is invariant when $a$ is an integer. The total contour integral is zero so that

$$
S[a ; x]=-n^{2} \operatorname{Res}_{z=-\frac{\log \sqrt{x}}{n}} \frac{1}{\sinh ^{2 a}\left(\frac{\log \sqrt{x}}{n}+z\right)} \operatorname{coth}(n z) .
$$

We use (2.7) to organize the answer. The two point function on $\mathcal{R}_{n}$ should proportional to a four point function with operators $W_{J}, W_{J}, \sigma_{n}, \tilde{\sigma}_{n}$. The same reason as (3.15) tells us

$$
\left\langle W_{J}\left(z_{1}\right) W_{J}\left(z_{2}\right)\right\rangle_{\mathcal{R}_{n}}=\frac{\mathcal{N}_{J}}{z_{12}^{2 J}} \sum_{j=0}^{J-1} b[J, j ; n] \xi^{j} .
$$

Note the maximal power of $\xi$ is $J-1$, this is just a direct consequence of the residue (A.6). We evaluate the residue (A.6) for the first few spins.It is enough to give the $n$ related constants $b[J, j ; n]$,

$$
\begin{aligned}
& b[3,0 ; n]=n, \quad b[3,1 ; n]=\frac{n^{2}-1}{4 n}, \quad b[3,2 ; n]=\frac{n^{4}-5 n^{2}+4}{120 n^{3}} \\
& b[4,0 ; n]=n, \quad b[4,1 ; n]=\frac{-1+n^{2}}{3 n}, \quad b[4,2 ; n]=\frac{7-10 n^{2}+3 n^{4}}{120 n^{3}}, \\
& b[4,3 ; n]=\frac{-36+49 n^{2}-14 n^{4}+n^{6}}{5040 n^{5}}, \quad b[5,0 ; n]=n, \quad b[5,1 ; n]=\frac{5\left(-1+n^{2}\right)}{12 n}, \\
& b[5,2 ; n]=\frac{13-20 n^{2}+7 n^{4}}{144 n^{3}}, \quad b[5,3 ; n]=\frac{-164+273 n^{2}-126 n^{4}+17 n^{6}}{12096 n^{5}}, \\
& b[5,4 ; n]=\frac{576-820 n^{2}+273 n^{4}-30 n^{6}+n^{8}}{362880 n^{7}}, \quad b[6,0 ; n]=n, \quad b[6,1 ; n]=\frac{-1+n^{2}}{2 n}, \\
& b[6,2 ; n]=\frac{31-50 n^{2}+19 n^{4}}{240 n^{3}}, \\
& b[6,3 ; n]=\frac{-695+1302 n^{2}-735 n^{4}+128 n^{6}}{30240 n^{5}}, \\
& b[6,4 ; n]=\frac{1916-3475 n^{2}+1953 n^{4}-425 n^{6}+31 n^{8}}{604800 n^{7}}, \\
& b[6,5 ; n]=\frac{-14400+21076 n^{2}-7645 n^{4}+1023 n^{6}-55 n^{8}+n^{10}}{39916800 n^{9}} .
\end{aligned}
$$

The terms with $j=0$ is always $n$, because these terms should contribute to the divergent terms (proportional to $\mathrm{M}$ ) and cancel with the terms coming from $n \log Z_{1}$. To simplify notation for Rényi entropy, we also introduce constants $\tilde{b}[J, j ; n]=\frac{b[J, j ; n]}{1-n}$ for $1 \leq j \leq J-1$,

$$
\begin{array}{llrl}
\tilde{b}[3,1 ; n] & =\frac{-1-n}{4 n}, & \tilde{b}[3,2 ; n] & =\frac{4+4 n-n^{2}-n^{3}}{120 n^{3}} \\
\tilde{b}[4,1 ; n] & =\frac{-1-n}{3 n}, & \tilde{b}[4,2 ; n] & =\frac{7+7 n-3 n^{2}-3 n^{3}}{120 n^{3}} \\
\tilde{b}[4,3 ; n] & =-\frac{36+36 n-13 n^{2}-13 n^{3}+n^{4}+n^{5}}{5040 n^{5}} & \\
\tilde{b}[5,1 ; n]=-\frac{5(1+n)}{12 n}, & \tilde{b}[5,2 ; n] & =-\frac{(1+n)\left(-13+7 n^{2}\right)}{144 n^{3}}
\end{array}
$$




$$
\begin{aligned}
& \tilde{b}[5,3 ; n]=-\frac{(1+n)\left(164-109 n^{2}+17 n^{4}\right)}{12096 n^{5}}, \\
& \tilde{b}[5,4 ; n]=-\frac{(1+n)\left(-576+244 n^{2}-29 n^{4}+n^{6}\right)}{362880 n^{7}} \\
& \tilde{b}[6,1 ; n]=-\frac{(1+n)}{2 n}, \quad \tilde{b}[6,2 ; n]=-\frac{(1+n)\left(-31+19 n^{2}\right)}{240 n^{3}}, \\
& \tilde{b}[6,3 ; n]=-\frac{(1+n)\left(695-607 n^{2}+128 n^{4}\right)}{30240 n^{5}} \\
& \tilde{b}[6,4 ; n]=-\frac{(1+n)\left(-1916+1559 n^{2}-394 n^{4}+31 n^{6}\right)}{604800 n^{7}}, \\
& \tilde{b}[6,5 ; n]=-\frac{(1+n)\left(14400-6676 n^{2}+969 n^{4}-54 n^{6}+n^{8}\right)}{39916800 n^{9}} .
\end{aligned}
$$

\section{A.2 Three point function on $\mathcal{R}_{n}$}

As in the previous subsection, we map $\mathcal{R}_{n}$ to complex plane,

$$
\begin{aligned}
\left\langle W_{J_{1}}\left(z_{1}\right) W_{J_{2}}\left(z_{2}\right) W_{J_{3}}\left(z_{3}\right)\right\rangle_{\mathcal{R}_{n}}= & \frac{C_{J_{1} J_{2} J_{3}}}{(2 n)^{J_{1}+J_{2}+J_{3}}} h\left[z_{1}\right]^{J_{1}} h\left[z_{2}\right]^{J_{2}} h\left[z_{3}\right]^{J_{3}} \times \\
& \times S\left[J_{1}+J_{2}-J_{3}, J_{2}+J_{3}-J_{1}, J_{3}+J_{1}-J_{2} ; r_{12}, r_{23}, r_{31}\right]
\end{aligned}
$$

Here

$$
h[z]=\frac{l_{12}}{\left(z-l_{1}\right)\left(z-l_{2}\right)}, \quad r_{i j}=\frac{\left(z_{i}-l_{1}\right)\left(z_{j}-l_{2}\right)}{\left(z_{i}-l_{2}\right)\left(z_{j}-l_{1}\right)}
$$

and $\mathrm{S}$ is a multi-summation,

$$
\begin{array}{cl}
S[a, b, c ; x, y, z]=\sum_{j_{1}, j_{2}, j_{3}=0}^{n-1} & \sinh ^{-a}\left(\frac{\log \sqrt{x}}{n}+\frac{\pi i\left(j_{1}-j_{2}\right)}{n}\right) \sinh ^{-b}\left(\frac{\log \sqrt{y}}{n}+\frac{\pi i\left(j_{2}-j_{3}\right)}{n}\right) \\
& \times \sinh ^{-c}\left(\frac{\log \sqrt{z}}{n}+\frac{\pi i\left(j_{3}-j_{1}\right)}{n}\right)
\end{array}
$$

This summation can be done similar to (A.3). In (A.8),

$$
r_{i j} r_{j k}=r_{i k}
$$

which is not the case for general $x, y, z$ in (A.10). So for the special summation in (A.8), the summation of $j_{3}$ is contributed by the poles at $\frac{\log \sqrt{r_{13}}}{n}+\frac{\pi i j_{1}}{n}$ and $\frac{\log \sqrt{r_{23}}}{n}+\frac{\pi i j_{2}}{n}$. This doesn't causing new poles. Then the $j_{2}$ summation is contributed from the poles at $\frac{\log \sqrt{r_{12}}}{n}+\frac{\pi i j_{1}}{n}$. The summation $j_{1}$ contributes a factor $\mathrm{n}$ as there is no pole now.

\section{A.3 Four point function on $\mathcal{R}_{n}$}

We only consider the case with four higher spin operators being the same.

$$
\begin{aligned}
\left\langle W_{J}\left(z_{1}\right) \cdots W_{J}\left(z_{4}\right)\right\rangle_{\mathcal{R}_{n}}= & \frac{\mathcal{N}_{J}^{2}}{(2 n)^{4 J}}\left(h\left[z_{1}\right] h\left[z_{2}\right] h\left[z_{3}\right] h\left[z_{4}\right]\right)^{J} \times \\
& \times \sum_{j=0}^{2 J} a[J, j] S\left[2 J-2 j, j, j, j, j, 2 J-2 j ; r_{12}, r_{13}, r_{14}, r_{23}, r_{24}, r_{34}\right],
\end{aligned}
$$


$S$ is defined as

$$
S\left[\left\{a_{i j}\right\} ;\left\{r_{i j}\right\} ; 1 \leq i<j \leq 4\right]=\sum_{j_{1}, j_{2}, j_{3}, j_{4}=0}^{n-1} \prod_{1 \leq k<l \leq 4} \sinh ^{-a_{k l}}\left(\frac{\log \sqrt{r_{k l}}}{n}+\frac{\pi i\left(j_{k}-j_{l}\right)}{n}\right) .
$$

This summation can be done using the residue theorem as before. In this work, we only need the four summations

$$
S[4,1,1,1,1,4], S[2,2,2,2,2,2], S[0,3,3,3,3,0], S[-2,4,4,4,4,-2] \text {, }
$$

each $S$ are quite lengthy. For the $\mathcal{O}(c)$ computation, we need the combination (6.15). As discussed in section 6.3.1, we can use the identity (6.16) to express (6.15) as $\mathcal{P}\left(y_{i j}\right)$

$$
\begin{aligned}
\mathcal{P}\left(y_{i j}\right) \equiv-\frac{1}{n^{4}}\left(S[4,1,1,1,1,4]-\frac{1}{10} S[2,2,2,2,2,2]\right. \\
+3 S[0,3,3,3,3,0]+S[-2,4,4,4,4,-2]) \\
=\sum_{\alpha=1}^{4} \sum_{a=1}^{2 \alpha+2} n^{1+2 \alpha} y_{12}^{a} \theta_{\alpha, a}\left(y_{i j}\right) .
\end{aligned}
$$

Similarly, to compute the quantum correction, we define

$$
\mathcal{Q}\left(y_{i j}\right) \equiv-\frac{1}{n^{4}} S[2,2,2,2,2,2]=\sum_{\alpha=1}^{4} \sum_{a=1}^{2 \alpha+2} n^{1+2 \alpha} y_{12}^{a} \psi_{\alpha, a}\left(y_{i j}\right) .
$$

The functions $\theta_{\alpha, a}$ are ${ }^{21}$

$$
\begin{aligned}
\theta_{1,1}= & -\frac{8192}{675}\left(3 d^{3}(e-h)+d\left(-5(-2+e) e+3 g-6 g^{2}+2(1-2 h) h\right)+f(e(3-6 f)\right. \\
& \left.+e^{2}(-2+4 f)+g(1-g+f(-5+3 f+3 g))+h+(1-3 f) f h+(-2+3 f) h^{2}\right) \\
& \left.+d^{2}\left(e(-10+3 e)-2 g+4 g^{2}+h(2+3 h)\right)\right), \\
\theta_{1,2}= & \frac{8192}{675}\left(3 d^{3}(e-h)-d\left(e(-25+9 e)+g(3+10 g)-3 h+6 h^{2}\right)+f\left(e^{2}(2+4 f)-e(3+10 f)\right.\right. \\
& \left.\left.+g(-2+3 g+3 f(f+g))-3 f^{2} h+3 f h^{2}\right)+d^{2}\left(3(-5+e) e+2 g+4 g^{2}+3 h(1+h)\right)\right), \\
\theta_{1,3}= & \frac{8192}{675}\left(d^{2}(5 e-4 g-h)+f\left(-4 e^{2}+4 e(3+f)-g(3+5 f+4 g)+h+f h-2 h^{2}\right)\right. \\
& +d(e(-21+4 e)+4 g(3+g)+h(-1+2 h))), \\
\theta_{1,4}= & \frac{16384}{225}(d-f)(e-g), \\
\theta_{2,1}= & \frac{1024}{225}\left(24 d^{5}(e-h)+f\left(50 e^{3}(1-2 f)+20 e^{4}(-1+2 f)+4 e^{2}(-1+2 f)(12+5(-1+f) f)\right.\right. \\
& +e(22-2 f(37+15 f(-3+2 f)))+g(5+f(-39+f(71+f(-65+24 f)))-15 g \\
& \left.+3 f(27+f(-25+9 f)) g+10(2+f(-8+3 f)) g^{2}+10(-1+3 f) g^{3}\right)+(4+f(4 \\
& +f(-20+(31-24 f) f))) h+3(-6+f(4+f(-2+9 f))) h^{2}-10(-3+f(2+3 f)) h^{3} \\
& \left.+10(-2+3 f) h^{4}\right)+d^{4}(e(-109+27 e)+20 g(-1+2 g)+h(35+27 h))+2 d(5 e(11 \\
& +e(-18-5(-3+e) e))+g(11+g(-37+15(3-2 g) g))+2 h(2+h(-9+5(3-2 h) h)))
\end{aligned}
$$

\footnotetext{
${ }^{21}$ To simplify notation, we define $d=y_{13}, e=y_{14}, f=y_{23}, g=y_{24}, h=y_{34}$.
} 


$$
\begin{aligned}
& +2 d^{2}(e(-105+e(114+5 e(-13+3 e)))+2 g(-1+2 g)(12+5(-1+g) g)+h(4 \\
& \left.+h(3+5 h(-1+3 h))))+d^{3}(3 e(69+e(-41+10 e))-2(25 g(-1+2 g)+h(11+3 h(2+5 h))))\right), \\
& \theta_{2,2}=-\frac{1024}{225}\left(24 d^{5}(e-h)+d^{4}(9 e(-17+3 e)+20 g(1+2 g)+3 h(13+9 h))-2 d(e(-400\right. \\
& +e(337+5 e(-34+9 e)))+g(11+g(131+5 g(-7+10 g)))+h(-1+2 h)(31+15(-1+h) h)) \\
& +2 d^{2}\left(e(-410+3 e(83+5(-6+e) e))+2 g(12+g(63+5 g(-1+2 g)))+h\left(31+15 h\left(2+h^{2}\right)\right)\right) \\
& +d^{3}(e(463+3 e(-57+10 e))-2(5 g(5+14 g)+3 h(14+h(3+5 h))))+f\left(20 e^{4}(1+2 f)\right. \\
& -10 e^{3}(5+14 f)+4 e^{2}(12+f(63+5 f(-1+2 f)))-2 e(11+f(131+5 f(-7+10 f))) \\
& +(-19+f(-35+f(55+3 f(-7+8 f)))) g+(53+3 f(19+9(-1+f) f)) g^{2}+10(-5 \\
& +3(-1+f) f) g^{3}+30(1+f) g^{4}+h\left(10-20 h+f\left(10-24 f^{3}+27 f^{2}(1+h)+6 h(13\right.\right. \\
& \left.\left.\left.\left.+5(-1+h) h)-6 f\left(13+5 h^{2}\right)\right)\right)\right)\right) \text {, } \\
& \theta_{2,3}=-\frac{1024}{225}\left(f \left(-40 e^{4}+40 e^{3}(4+f)-2 e^{2}(199+4 f(34+5 f))+e(568+2 f(291+20 f(2+f)))\right.\right. \\
& -g\left(33+44 f^{3}+8 f^{2}(13+6 g)+g(203+10 g(-1+4 g))+f(109+g(99+50 g))\right)+2(12 \\
& \left.+f(12+f(59+2 f))) h-2(29+3 f(21+f)) h^{2}+10(3+f) h^{3}-20 h^{4}\right)+d^{4}(44 e-4(10 g+h)) \\
& +2 d^{3}(4 e(-47+6 e)+20 g(4+g)+h(61+3 h))+d\left(e\left(-2259+5 e\left(211-50 e+8 e^{2}\right)\right)\right. \\
& +2 g(284+g(291+20 g(2+g)))+2 h(-1+2 h)(72+5(-1+h) h))+d^{2}(e(1321 \\
& +e(-393+50 e))-2(g(199+4 g(34+5 g))+h(72+h(57+5 h))))) \text {, } \\
& \theta_{2,4}=\frac{1024}{225}\left(60 d^{3}(-2 e+g+h)+f\left(60 e^{3}-2 e^{2}(267+68 f)+2 e(742+f(313+30 f))-g(513\right.\right. \\
& \left.\left.+f(377+120 f)+367 g+123 f g+60 g^{2}\right)+10(7+f(7+6 f)) h-20(7+3 f) h^{2}\right)+d(e(-3033 \\
& +(793-60 e) e)+2 g(742+g(313+30 g))+130 h(-1+2 h))-d^{2}(e(-983+123 e) \\
& +2 g(267+68 g)+10 h(13+6 h))), \\
& \theta_{2,5}=-\frac{8192}{225}\left(d^{2}(34 e-29 g-5 h)+f\left(-29 e^{2}+e(180+29 f)-g(117+34 f+29 g)\right.\right. \\
& \left.\left.+5(1+f) h-10 h^{2}\right)+d(e(-243+29 e)+g(180+29 g)+5 h(-1+2 h))\right), \\
& \theta_{2,6}=-\frac{32768}{15}(d-f)(e-g), \\
& \theta_{3,1}=-\frac{256}{225}\left(120 d^{5}(e-h)+f\left(-4 e(-1+2 f)(17+75(-1+f) f)+4 e^{2}(-1+2 f)(53+175(-1+f) f)\right.\right. \\
& -250 e^{3}\left(-1+f\left(5-9 f+6 f^{2}\right)\right)+100 e^{4}\left(-1+f\left(5-9 f+6 f^{2}\right)\right)+g(11+f(-125+f(313 \\
& \left.+5 f(-65+24 f)))-61 g+3 f(121+5 f(-25+9 f)) g+50(2+f(-8+3 f)) g^{2}+50(-1+3 f) g^{3}\right) \\
& +(6+f(6+f(-58+5(31-24 f) f))) h+(-62+3 f(6+5 f(-2+9 f))) h^{2}-50(-3+f(2 \\
& \left.+3 f)) h^{3}+50(-2+3 f) h^{4}\right)+5 d^{4}\left(e(-109+27 e)+20 g\left(-1+g\left(5-9 g+6 g^{2}\right)\right)+h(35+27 h)\right) \\
& +d^{3}\left(3 e(331+5 e(-41+10 e))-250 g\left(-1+g\left(5-9 g+6 g^{2}\right)\right)-2 h(34+15 h(2+5 h))\right) \\
& +2 d(-5 e(-41+e(83+25(-3+e) e))-2 g(-1+2 g)(17+75(-1+g) g)+2 h(3 \\
& +h(-31+25(3-2 h) h)))+2 d^{2}(e(-455+e(549+25 e(-13+3 e)))+2 g(-1+2 g)(53 \\
& +175(-1+g) g)+h(6+h(-6+25 h(-1+3 h))))) \text {, } \\
& \theta_{3,2}=\frac{256}{225}\left(840 d^{5}(e-h)+5 d^{4}\left(3 e(-313+63 e)+20 g\left(1+g\left(11-3 g+6 g^{2}\right)\right)+9 h(29+21 h)\right)\right. \\
& +2 d^{2}(e(-7045+3 e(2013+25 e(-37+7 e)))+2 g(53+g(1111+25 g(-31+38 g)))+h(134
\end{aligned}
$$




$$
\begin{aligned}
& +3 h(-1+5 h)(2+35 h)))+d^{3}(e(11063+15 e(-351+70 e))-50 g(5+g(71+3 g(-9 \\
& +14 g)))-6 h(118+5 h(18+35 h)))-2 d(e(-4975+e(6557+25 e(-181+51 e)))+2(g(17 \\
& +25 g(27+g(-35+38 g)))+h(-1+2 h)(67+225(-1+h) h)))+f\left(100 e^{4}(1\right. \\
& \left.+f\left(11-3 f+6 f^{2}\right)\right)-50 e^{3}(5+f(71+3 f(-9+14 f)))-4 e(17+25 f(27+f(-35+38 f))) \\
& +4 e^{2}(53+f(1111+25 f(-31+38 f)))+(-67+f(-235+f(863+15 f(-93+56 f)))) g \\
& +(373+9 f(117+5 f(-37+21 f))) g^{2}+50(-14+3 f(-12+7 f)) g^{3}+150(3+7 f) g^{4} \\
& +h\left(-840 f^{4}+15 f^{3}(67+63 h)-6 f^{2}(93+5 h(3+35 h))-50\left(-1+h\left(5-9 h+6 h^{2}\right)\right)\right. \\
& +2 f(25+3 h(73+25 h(-6+7 h)))))), \\
& \theta_{3,3}=-\frac{256}{225}\left(1440 d^{5}(e-h)+f\left(100 e^{4}(23+3 f(7+2 f))-50 e^{3}(163+f(169+12 f(3+f)))\right.\right. \\
& +2 e^{2}(5902+5 f(1402+5 f(37+78 f)))-2 e(4486+f(6122+25 f(-7+166 f))) \\
& +g(123+2339 g+5(f(119+4 f(29+2 f(7+36 f)))+3 f(37+4 f(1+27 f)) g \\
& \left.\left.+10(-5+4 f)(11+9 f) g^{2}+40(16+9 f) g^{3}\right)\right)-2(53+f(53+5 f(185+4 f(-37+36 f)))) h \\
& \left.+2(281+15 f(71+f(7+54 f))) h^{2}-50(21+f(43+36 f)) h^{3}+100(7+18 f) h^{4}\right) \\
& +20 d^{4}(e(-536+81 e)+5 g(23+3 g(7+2 g))+h(124+81 h))-d(e(-66441+e(58559 \\
& +850 e(-35+8 e)))+2 g(4486+g(6122+25 g(-7+166 g)))+2 h(-1+2 h)(833 \\
& +1075(-1+h) h))+10 d^{3}\left(2 e\left(1729-597 e+90 e^{2}\right)-5 g(163+g(169+12 g(3\right. \\
& +g)))-h(235+3 h(43+60 h)))+d^{2}(5 e(-12515+e(7461+10 e(-251+36 e))) \\
& +2(g(5902+5 g(1402+5 g(37+78 g)))+h(833+5 h(63+5 h(7+36 h)))))), \\
& \theta_{3,4}=\frac{256}{225}\left(720 d^{5}(e-h)+30 d^{4}(e(-307+27 e)+50 g(3+g)+h(53+27 h))-d(e(-204081\right. \\
& +5 e(23851+30 e(-275+46 e)))+2 g(28238+5 g(3826+15 g(83+50 g)))+10 h(-1 \\
& +2 h)(449+195(-1+h) h))+30 d^{3}\left(e\left(1553-339 e+30 e^{2}\right)-5 g(13+2 g)(11+3 g)-h(125\right. \\
& \left.\left.+39 h+30 h^{2}\right)\right)+f\left(1500 e^{4}(3+f)-150 e^{3}(13+2 f)(11+3 f)+10 e^{2}(4614+f(2546\right. \\
& +15 f(39+10 f)))-2 e(28238+5 f(3826+15 f(83+50 f)))+(8781+5 f(1601 \\
& +6 f(193+f(133+24 f)))) g+5(1969+3 f(371+6 f(47+9 f))) g^{2}+150(15+f(29 \\
& +6 f)) g^{3}+300(17+3 f) g^{4}+10 h(-131-f(131+3 f(105+f(-13+24 f)))+367 h+3 f(133 \\
& \left.\left.+3 f(7+9 f)) h-15(21+f(13+6 f)) h^{2}+30(7+3 f) h^{3}\right)\right)+5 d^{2}(e(-25499+3 e(3311 \\
& +10 e(-71+6 e)))+2 g(4614+g(2546+15 g(39+10 g)))+2 h(449+3 h(73+5 h(7+6 h))))), \\
& \theta_{3,5}=\frac{1024}{45}\left(12 d^{4}(11 e-10 g-h)+f\left(-120 e^{4}+30 e^{3}(33+5 f)-e^{2}(3649+3 f(369+50 f))\right.\right. \\
& +e(7353+f(3163+30 f(27+4 f)))-2 g(1188+f(715+6 f(53+11 f))+707 g \\
& \left.+36 f(9+2 f) g+15(17+5 f) g^{2}+60 g^{3}\right)+2\left(77+f\left(77+69 f+6 f^{2}\right)\right) h-2(169+9 f(9 \\
& \left.+f)) h^{2}+30(3+f) h^{3}-60 h^{4}\right)+6 d^{3}\left(-242 e+24 e^{2}+5 g(33+5 g)+h(25+3 h)\right) \\
& +d(2 e(-8433+e(3146+15 e(-43+4 e)))+g(7353+g(3163+30 g(27+4 g))) \\
& +2 h(-1+2 h)(149+15(-1+h) h))+d^{2}(10 e(674+3 e(-51+5 e))-g(3649 \\
& +3 g(369+50 g))-2 h(149+3 h(21+5 h)))),
\end{aligned}
$$




$$
\begin{aligned}
& \theta_{3,6}=\frac{1024}{15}\left(4 d^{3}(29 e-25 g-4 h)+d(5 e(1037+5 e(-45+4 e))-g(3169+g(827+100 g))\right. \\
& +64(1-2 h) h)+f\left(-100 e^{3}+e^{2}(881+123 f)-e(3169+f(827+100 f))+g(1657\right. \\
& \left.\left.+f(615+116 f)+583 g+121 f g+100 g^{2}\right)-16(3+f(3+f)) h+16(6+f) h^{2}\right) \\
& \left.+d^{2}(e(-1205+121 e)+g(881+123 g)+16 h(4+h))\right) \text {, } \\
& \theta_{3,7}=\frac{4096}{15}\left(d^{2}(65 e-61 g-4 h)+f\left(-61 e^{2}+e(504+61 f)-g(378+65 f+61 g)\right.\right. \\
& \left.\left.+4(1+f) h-8 h^{2}\right)+d\left(e(-630+61 e)+g(504+61 g)-4 h+8 h^{2}\right)\right), \\
& \theta_{3,8}=\frac{172032}{5}(d-f)(e-g) \text {, } \\
& \theta_{4,1}=\frac{256}{675}\left(72 d^{5}(e-h)+f\left(-36 e(-1+2 f)(1+15(-1+f) f)+124 e^{2}(-1+2 f)(1+15(-1+f) f)\right.\right. \\
& -150 e^{3}(-1+2 f)(1+15(-1+f) f)+60 e^{4}(-1+2 f)(1+15(-1+f) f)+g(5+f(-67 \\
& +3 f(61+f(-65+24 f)))-35 g+3 f(71+3 f(-25+9 f)) g+30(2+f(-8+3 f)) g^{2} \\
& \left.+30(-1+3 f) g^{3}\right)+(2+f(2-3 f(-2+3 f)(-5+8 f))) h+(-34+3 f(2+3 f(-2+9 f))) h^{2} \\
& \left.-30(-3+f(2+3 f)) h^{3}+30(-2+3 f) h^{4}\right)+3 d^{4}(e(-109+27 e)+20 g(-1+2 g)(1 \\
& +15(-1+g) g)+h(35+27 h))-2 d(5 e(-23+e(49+15(-3+e) e))+18 g(-1+2 g)(1 \\
& +15(-1+g) g)+2 h(-1+2 h)(1+15(-1+h) h))+2 d^{2}(e(-265+3 e(109+5 e(-13+3 e))) \\
& \left.+62 g(-1+2 g)(1+15(-1+g) g)+h(-1+3 h)\left(-2+15 h^{2}\right)\right)+3 d^{3}(e(197+3 e(-41 \\
& +10 e))-50 g(-1+2 g)(1+15(-1+g) g)-6 h(2+h(2+5 h)))) \text {, } \\
& \theta_{4,2}=-\frac{256}{675}\left(2232 d^{5}(e-h)+3 d^{4}\left(-4083 e+837 e^{2}+20 g(1+g(47+15 g(-13+14 g)))\right.\right. \\
& +3 h(383+279 h))-2 d(e(-10525+3 e(5257+5 e(-769+219 e)))+2(g(9+g(1319 \\
& +15 g(-341+350 g)))+39 h(-1+2 h)(1+15(-1+h) h)))+2 d^{2}(3 e(-5485+e(5059 \\
& +15 e(-161+31 e)))+2 g(31+g(2609+15 g(-691+722 g)))+3 h(26+5 h(-22 \\
& +3 h(-5+31 h))))+3 d^{3}(3 e(3081+e(-1527+310 e))-2(5 g(5+g(299+15 g(-81+86 g))) \\
& +h(202+3 h(78+155 h))))+f\left(60 e^{4}(1+f(47+15 f(-13+14 f)))-30 e^{3}(5+f(299\right. \\
& +15 f(-81+86 f)))-4 e(9+f(1319+15 f(-341+350 f)))+4 e^{2}(31+f(2609 \\
& +15 f(-691+722 f)))+(-37+3 f(-95+3 f(225+f(-437+248 f)))) g+3(193 \\
& +f(857+9 f(-173+93 f))) g^{2}+30(-50+3 f(-56+31 f)) g^{3}+90(11+31 f) g^{4} \\
& +3 h\left(-744 f^{4}+f^{3}(897+837 h)-2 f^{2}(139+15 h(3+31 h))+10(1+h(-17+15(3-2 h) h))\right. \\
& +2 f(5+h(79+15 h(-26+31 h)))))) \text {, } \\
& \theta_{4,3}=\frac{256}{675}\left(12960 d^{5}(e-h)+f\left(60 e^{4}\left(107+15 f\left(7+2 f+24 f^{2}\right)\right)-30 e^{3}(751+f(829\right.\right. \\
& +60 f(-3+49 f)))-2 e(10242+f(14938+15 f(-1147+3694 f)))+2 e^{2}(15382 \\
& +3 f(6466+5 f(-911+4710 f)))+g(69+f(637+12 f(-49+4 f(-151+270 f))) \\
& +4709 g+9 f(53+4 f(-281+405 f)) g+30(-631+5 f(-77+108 f)) g^{2}+120(166 \\
& \left.+135 f) g^{3}\right)-2(31+f(31+3 f(689+16 f(-148+135 f)))) h+2(527+9 f(271 \\
& \left.+f(31+810 f))) h^{2}-30(93+f(571+540 f)) h^{3}+60(31+270 f) h^{4}\right)+12 d^{4}(e(-7226 \\
& \left.+1215 e)+5 g\left(107+15 g\left(7+2 g+24 g^{2}\right)\right)+h(1786+1215 h)\right)+6 d^{3}(2 e(20419 \\
& +3 e(-2689+450 e))-5 g(751+g(829+60 g(-3+49 g)))-h(1291+3 h(571
\end{aligned}
$$




$$
\begin{aligned}
& +900 h)))-d(e(-331647+5 e(73453+6 e(-7195+1744 e)))+2(g(10242+g(14938 \\
& +15 g(-1147+3694 g)))+571 h(-1+2 h)(1+15(-1+h) h)))+d^{2}(e(-376153 \\
& +3 e(88653+50 e(-679+108 e)))+2(g(15382+3 g(6466+5 g(-911+4710 g))) \\
& +h(571+3 h(-993+5 h(31+540 h)))))) \text {, } \\
& \theta_{4,4}=-\frac{256}{225}\left(9360 d^{5}(e-h)+30 d^{4}(e(-2671+351 e)+10 g(51+g(41+12 g(3+g)))\right. \\
& +h(569+351 h))+f\left(300 e^{4}(51+f(41+12 f(3+f)))-30 e^{3}(2147+5 f(349+42 f(7\right. \\
& +4 f)))+2 e^{2}(56022+f(46478+75 f(471+442 f)))-2 e(51762+f(44338+15 f(1847 \\
& +2890 f)))+(6793+f(6497+30 f(189+f(409+312 f)))) g+(8377+3 f(1691 \\
& +30 f(131+117 f))) g^{2}+30(-173+5 f(77+78 f)) g^{3}+300(101+39 f) g^{4} \\
& +10 h\left(-31-f(31+3 f(73+f(-289+312 f)))+527 h+3 f\left(197+93 f+351 f^{2}\right) h-15(93\right. \\
& \left.\left.+f(109+78 f)) h^{2}+30(31+39 f) h^{3}\right)\right)+30 d^{3}(e(9709+3 e(-989+130 e))-g(2147 \\
& +5 g(349+42 g(7+4 g)))-3 h(71+h(109+130 h)))-d(e(-698533+e(577243 \\
& +30 e(-8927+1790 e)))+2(g(51762+g(44338+15 g(1847+2890 g)))+545 h(-1 \\
& +2 h)(1+15(-1+h) h)))+d^{2}(e(-584723+3 e(104591+50 e(-623+78 e))) \\
& +2(g(56022+g(46478+75 g(471+442 g)))+5 h(109+3 h(-223+5 h(31+78 h)))))), \\
& \theta_{4,5}=\frac{1024}{225}\left(2160 d^{5}(e-h)+30 d^{4}(e(-877+81 e)+20 g(16+3 g(3+g))+h(155+81 h))\right. \\
& -d(2 e(-288783+e(176264+75 e(-829+130 e)))+g(148725+g(98743+150 g(375 \\
& +172 g)))+370 h(-1+2 h)(1+15(-1+h) h))+f\left(600 e^{4}(16+3 f(3+f))-150 e^{3}(339\right. \\
& +f(203+6 f(15+2 f)))+e^{2}(116293+75 f(981+2 f(257+72 f)))-e(148725 \\
& +f(98743+150 f(375+172 f)))+2(g(12087+f(10054+15 f(553+f(355+72 f))) \\
& \left.+10034 g+45 f(183+f(125+27 f)) g+75(101+f(77+18 f)) g^{2}+150(47+9 f) g^{3}\right) \\
& -5(19+f(19+3 f(5+f(-43+72 f)))) h+5(323+9 f(27+f(19+27 f))) h^{2}-75(57 \\
& \left.\left.+f(37+18 f)) h^{3}+150(19+9 f) h^{4}\right)\right)+30 d^{3}\left(e\left(4361-969 e+90 e^{2}\right)-5 g(339\right. \\
& +g(203+6 g(15+2 g)))-h(61+3 h(37+30 h)))+d^{2}(2 e(-177364+75 e(933 \\
& +e(-203+18 e)))+g(116293+75 g(981+2 g(257+72 g)))+10 h(37 \\
& \left.\left.\left.+3 h\left(-87+95 h+90 h^{2}\right)\right)\right)\right) \text {, } \\
& \theta_{4,6}=-\frac{1024}{15}\left(48 d^{5}(e-h)+f\left(60 e^{4}(11+3 f)-100 e^{3}(49+3 f(6+f))+e^{2}(15749\right.\right. \\
& +3 f(2309+60 f(11+f)))-e(28589+f(14183+20 f(266+51 f)))+g(8303 \\
& +f(5277+2 f(1351+3 f(125+8 f)))+5245 g+f(2767+54 f(15+f)) g \\
& \left.+20(137+3 f(14+f)) g^{2}+60(13+f) g^{3}\right)-2(6+f(6+f(-10+3 f(3+8 f)))) h \\
& \left.+2(102+f(62+27 f(2+f))) h^{2}-60(9+f(4+f)) h^{3}+60(6+f) h^{4}\right)+6 d^{4}(3 e(-61+3 e) \\
& +10 g(11+3 g)+h(25+9 h))-d(e(-74453+25 e(1305+4 e(-82+9 e)))+g(28589 \\
& +g(14183+20 g(266+51 g)))+16 h(-1+2 h)(1+15(-1+h) h))+2 d^{3}(e(4207-603 e \\
& \left.\left.+30 e^{2}\right)-50 g(49+3 g(6+g))-2 h(16+3 h(12+5 h))\right)+d^{2}(e(-32705+e(8941 \\
& +60(-21+e) e))+g(15749+3 g(2309+60 g(11+g)))+4 h(4+h(-32+15 h(3+h))))),
\end{aligned}
$$




$$
\begin{aligned}
\theta_{4,7}= & -\frac{2048}{15}\left(12 d^{4}(11 e-10 g-h)+6 d^{3}\left(12 e(-29+2 e)+5 g(55+9 g)+h+3 h^{2}\right)\right. \\
& +f\left(-120 e^{4}+30 e^{3}(55+9 f)-2 e^{2}(4219+27 f(42+5 f))+2 e(11466+f(3967\right. \\
& +15 f(57+4 f)))-g\left(10143+132 f^{3}+24 f^{2}(53+6 g)+g(4469+30 g(43+4 g))\right. \\
& +f(4477+3 g(441+50 g)))+2\left(1+f-3 f^{2}+6 f^{3}\right) h-2(17+9 f(1+f)) h^{2}+30(3 \\
& \left.+f) h^{3}-60 h^{4}\right)+d(e(-43785+e(13037+30 e(-69+4 e)))+2 g(11466+g(3967 \\
& +15 g(57+4 g)))+2 h(-1+2 h)(1+15(-1+h) h))+d^{2}(5 e(2609-441 e \\
& \left.\left.\left.+30 e^{2}\right)-2\left(g(4219+27 g(42+5 g))+h-9 h^{2}+15 h^{3}\right)\right)\right), \\
\theta_{4,8}= & \frac{14336}{5}\left(20 d^{3}(-e+g)-3 d^{2}(e(-89+7 e)+g(73+9 g))+f\left(20 e^{3}-3 e^{2}(73+9 f)\right.\right. \\
& \left.+e(993+f(213+20 f))-g\left(609+20 f^{2}+5 g(33+4 g)+3 f(55+7 g)\right)\right)+d(e(-1473 \\
& +(267-20 e) e)+g(993+g(213+20 g)))), \\
\theta_{4,9}= & \frac{68128}{5}\left(d^{2}(-e+g)-d((-12+e) e+g(10+g))+f\left(e^{2}-e(10+f)+g(8+f+g)\right)\right), \\
\theta_{4,10}= & -\frac{1376256}{5}(d-f)(e-g) .
\end{aligned}
$$

The functions $\psi_{\alpha, a}$ are

$$
\begin{aligned}
& \psi_{1,1}=\frac{256}{315}\left(52 d^{3}(-e+h)+d(70(-2+e) e+27 g(-1+2 g)+43 h(-1+2 h))-d^{2}(e(-165\right. \\
& +52 e)+18 g(-1+2 g)+h(43+52 h))-f\left(e(27-54 f)+18 e^{2}(-1+2 f)+(34\right. \\
& \left.\left.+f(-95+52 f)) g+(-34+52 f) g^{2}+h(9-18 h+f(9-52 f+52 h))\right)\right), \\
& \psi_{1,2}=\frac{256}{315}\left(52 d^{3}(e-h)+f\left(18 e^{2}(1+2 f)-9 e(3+10 f)+g(2(-9+g)+f(-25+52 f\right.\right. \\
& \left.+52 g))-(25+f(25+52 f)) h+2(25+26 f) h^{2}\right)-d(2 e(-150+53 e)+9 g(3+10 g) \\
& \left.+77 h(-1+2 h))+d^{2}(e(-235+52 e)+18 g(1+2 g)+h(77+52 h))\right), \\
& \psi_{1,3}=\frac{512}{315}\left(d^{2}(35 e-18 g-17 h)+f\left(-18 e^{2}+18 e(3+f)-g(1+35 f+18 g)+17(1\right.\right. \\
& \left.\left.+f) h-34 h^{2}\right)+d(e(-107+18 e)+18 g(3+g)+17 h(-1+2 h))\right), \\
& \psi_{1,4}=\frac{1536}{35}(d-f)(e-g) \\
& \psi_{2,1}=\frac{64}{45}\left(192 d^{5}(e-h)+3 d(-30(-2+e) e+7(1-2 g) g+23(1-2 h) h)+96 d^{4}((-7+e) e\right. \\
& +h(5+h))-4 d^{3}(e(-219+56 e)+h(99+64 h))+d^{2}(3 e(-185+68 e)+14 g(-1+2 g) \\
& +h(69+268 h))+f\left(e(21-42 f)+14 e^{2}(-1+2 f)+(30+f(-177+4 f(107+24 f(-5\right. \\
& +2 f))) g+2(-15+2 f(27+8 f(-5+3 f))) g^{2}+h(7-14 h \\
& +f(7+76 h+4 f(-35-32 h+24 f(3-2 f+h)))))), \\
& \psi_{2,2}=\frac{64}{45}\left(192 d^{5}(-e+h)+f\left(-14 e^{2}(1+14 f)+7 e(3+58 f)+g(-18+66 g+f(255-276 g\right.\right. \\
& +4 f(-85+24 g-24 f(-3+2 f+g))))+(55+f(55+4 f(61+24 f(-1+2 f)))) h \\
& \left.-2\left(55+122 f+48 f^{3}\right) h^{2}\right)-96 d^{4}((-9+e) e+h(7+h))+d(6 e(-250+97 e) \\
& +7 g(3+58 g)+491 h(-1+2 h))+4 d^{3}(e(-421+72 e)+h(253+96 h))-d^{2}(3 e(-695 \\
& +188 e)+14 g(1+14 g)+h(491+820 h))) \text {, } \\
& \psi_{2,3}=\frac{128}{45}\left(96 d^{4}(-e+h)+f\left(14 e^{2}(13+12 f)-14 e(33+37 f)+g(-33+102 g+f(8 f(17\right.\right. \\
& \left.+12 f+4 g)+27(3+8 g)))-(211+f(211+8 f(29+12 f))) h+2(211+4 f(33+8 f)) h^{2}\right)
\end{aligned}
$$




$$
\begin{aligned}
& -d(3 e(-727+210 e)+14 g(33+37 g)+571 h(-1+2 h))-8 d^{3}(e(-73+4 e)+h(61+8 h)) \\
& \left.+d^{2}(3 e(-563+104 e)+14 g(13+12 g)+h(571+456 h))\right) \text {, } \\
& \psi_{2,4}=\frac{128}{15}\left(60 d^{3}(-e+h)+f\left(-28 e^{2}(4+f)+7 e(61+24 f)-g(99+96 g+4 f(39+15 f+11 g))\right.\right. \\
& \left.+60(2+f(2+f)) h-60(4+f) h^{2}\right)-4 d^{2}(e(-106+11 e)+7 g(4+g)+15 h(3+h)) \\
& +d(e(-959+184 e)+7 g(61+24 g)+180 h(-1+2 h))), \\
& \psi_{2,5}=\frac{512}{15}\left(d^{2}(-29 e+14 g+15 h)+f\left(14 e^{2}-7 e(15+2 f)+g(62+29 f+14 g)-15(1+f) h\right.\right. \\
& \left.\left.+30 h^{2}\right)-d(2 e(-74+7 e)+7 g(15+2 g)+15 h(-1+2 h))\right), \\
& \psi_{2,6}=-\frac{3584}{3}(d-f)(e-g), \\
& \psi_{3,1}=\frac{128}{45}\left(120 d^{5}(-e+h)+f\left(e^{2}(2-4 f)+e(-3+6 f)+g(6(-1+g)+f(75-48 g\right.\right. \\
& -4 f(62-25 g+15 f(-5+2 f+g))))+(-1+f(-1+4 f(17+15 f(-3+2 f)))) h \\
& \left.+2(1-2 f(7+5 f(-4+3 f))) h^{2}\right)-60 d^{4}((-7+e) e+h(5+h))+3 d(10(-2+e) e \\
& +g(-1+2 g)+9 h(-1+2 h))+4 d^{3}(e(-132+35 e)+h(57+40 h))-d^{2}(3 e(-95 \\
& \left.\left.+36 e)-2 g+4 g^{2}+h(27+148 h)\right)\right) \text {, } \\
& \psi_{3,2}=\frac{128}{45}\left(840 d^{5}(e-h)-d(6 e(-250+99 e)+g(3+250 g)+653 h(-1+2 h))+60 d^{4}(e(-57\right. \\
& +7 e)+h(43+7 h))-4 d^{3}(e(-1312+285 e)+h(667+360 h))+d^{2}(27 e(-145+44 e) \\
& +2 g(1+62 g)+h(653+1948 h))+f\left(2 e^{2}(1+62 f)-e(3+250 f)+(18+f(-285\right. \\
& +4 f(262+15 f(-27+14 f)))) g+6(-7+2 f(24+5 f(-9+7 f))) g^{2}+h(-25+50 h \\
& +f(-25+148 h-4 f(67+15 f(-13+14 f-7 h)+60 h))))), \\
& \psi_{3,3}=\frac{256}{45}\left(720 d^{5}(-e+h)+20 d^{3}\left(e(-367+61 e)+220 h+86 h^{2}\right)+f\left(-2 e^{2}(121+180 f)\right.\right. \\
& +e(606+962 f)+g(21-72 g-5 f(-3+78 g+4 f(17-11 g+3 f(-11+12 f+6 g)))) \\
& \left.+\left(313+f\left(313+20 f\left(20+3 f+36 f^{2}\right)\right)\right) h-2(313+10 f(27+2 f(7+9 f))) h^{2}\right) \\
& +d\left(3 e(-1731+544 e)+606 g+962 g^{2}+1993 h(-1+2 h)\right)-60 d^{4}(e(-61+6 e) \\
& \left.+h(49+6 h))-d^{2}\left(15 e(-535+126 e)+242 g+360 g^{2}+h(1993+3540 h)\right)\right), \\
& \psi_{3,4}=\frac{256}{15}\left(120 d^{5}(e-h)+f\left(20 e^{2}(22+13 f)-e(1441+960 f)+g(9(19+30 g)+10 f(33\right.\right. \\
& +29 g+2 f(7+4 g+3 f(4+2 f+g))))-20(28+f(28+f(25+6 f(3+f)))) h+20(56 \\
& \left.+f(32+f(14+3 f))) h^{2}\right)+60 d^{4}((-16+e) e+h(14+h))-d(e(-5471+1330 e) \\
& +g(1441+960 g)+1740 h(-1+2 h))-20 d^{3}(e(-147+16 e)+h(105+26 h)) \\
& \left.+10 d^{2}(e(-517+89 e)+2(g(22+13 g)+h(87+92 h)))\right), \\
& \psi_{3,5}=\frac{1024}{3}\left(12 d^{4}(e-h)+f\left(-4 e^{2}(11+3 f)+2 e(99+34 f)-g(61+34 g+f(55+18 g\right.\right. \\
& \left.+2 f(13+6 f+2 g)))+(59+f(59+2 f(19+6 f))) h-2(59+f(21+4 f)) h^{2}\right) \\
& +d(e(-461+82 e)+2 g(99+34 g)+113 h(-1+2 h))+d^{3}\left(-82 e+4 e^{2}+2 h(35+4 h)\right) \\
& \left.-d^{2}(5 e(-53+6 e)+4 g(11+3 g)+h(113+66 h))\right) \text {, } \\
& \psi_{3,6}=\frac{2048}{3}\left(9 d^{3}(e-h)+f\left(e^{2}(19+2 f)-e(131+23 f)+g(17(4+g)+f(35+9 f+4 g))\right.\right. \\
& \left.-9(3+f(3+f)) h+9(6+f) h^{2}\right)+d^{2}\left(-70 e+4 e^{2}+g(19+2 g)+9 h(4+h)\right)-d(5 e(-43 \\
& +5 e)+g(131+23 g)+36 h(-1+2 h))) \text {, }
\end{aligned}
$$




$$
\begin{aligned}
& \psi_{3,7}=2048\left(d^{2}(5 e-2 g-3 h)+f\left(-2 e^{2}+2 e(14+f)-g(21+5 f+2 g)+3(1+f) h-6 h^{2}\right)\right. \\
& \left.+d\left(e(-35+2 e)+2 g(14+g)-3 h+6 h^{2}\right)\right), \\
& \psi_{3,8}=14336(d-f)(e-g), \\
& \psi_{4,1}=\frac{64}{315}\left(336 d^{5}(e-h)+d\left(-70(-2+e) e+3 g-6 g^{2}+67(1-2 h) h\right)+f(e(3-6 f)\right. \\
& +e^{2}(-2+4 f)+g(-10(-1+g)+f(-191+124 g+4 f(171-70 g+42 f(-5+2 f+g)))) \\
& \left.+h+f(1-12 f(15+14 f(-3+2 f))) h+2(-1+2 f(17+14 f(-4+3 f))) h^{2}\right) \\
& +168 d^{4}((-7+e) e+h(5+h))-4 d^{3}(e(-367+98 e)+h(157+112 h))+d^{2}(e(-765 \\
& \left.\left.+292 e)-2 g+4 g^{2}+h(67+404 h)\right)\right) \text {, } \\
& \psi_{4,2}=\frac{64}{315}\left(-10416 d^{5}(e-h)+d(2 e(-5850+2333 e)+g(3+1018 g)+6013 h(-1+2 h))\right. \\
& -168 d^{4}(e(-249+31 e)+h(187+31 h))+4 d^{3}(e(-15473+3486 e)+h(7619+4368 h)) \\
& -d^{2}\left(e(-41155+12892 e)+2 g+508 g^{2}+h(6013+21740 h)\right)+f\left(-2 e^{2}(1+254 f)\right. \\
& +e(3+1018 f)+(-54+f(2305-4 f(3125+42 f(-123+62 f)))) g+(118-4 f(577 \\
& +42 f(-41+31 f))) g^{2}+h(65-130 h+f(65-572 h+4 f(563+42 f(-61 \\
& +62 f-3(h)+840 h))))) \text {, } \\
& \psi_{4,3}=\frac{128}{315}\left(30240 d^{5}(e-h)+f\left(e^{2}(2186+3864 f)-2 e(2733+4957 f)+g(-59+366 g\right.\right. \\
& +7 f(-91+564 g+8 f(248-239 g+3 f(-239+180 f+90 g))))-(2973+f(2973 \\
& \left.+56 f(71+3 f(-59+180 f)))) h+2(2973+56 f(51+f(31+135 f))) h^{2}\right)-d(e(-88103 \\
& \left.+28590 e)+5466 g+9914 g^{2}+44133 h(-1+2 h)\right)+168 d^{4}(e(-841+90 e)+h(661+90 h)) \\
& -56 d^{3}(e(-4462+841 e)+h(2479+1142 h))+d^{2}(7 e(-30221+7788 e)+2186 g \\
& \left.\left.+3864 g^{2}+3 h(14711+35616 h)\right)\right) \text {, } \\
& \psi_{4,4}=\frac{128}{105}\left(-21840 d^{5}(e-h)+f\left(-84 e^{2}(104+81 f)+e(27301+22344 f)+g(-3(579+1036 g)\right.\right. \\
& +28 f(-2(63+67 g)+5 f(13+16 g-6 f(-8+26 f+13 g))))+140(98+f(98+f(95 \\
& \left.+12 f(9+13 f)))) h-140(196+f(157+2 f(62+39 f))) h^{2}\right)+d(e(-180797+47572 e) \\
& +g(27301+22344 g)+83580 h(-1+2 h))-840 d^{4}(e(-148+13 e)+h(122+13 h)) \\
& +140 d^{3}(e(-1947+296 e)+h(1215+436 h))-28 d^{2}(e(-10759+2234 e)+3 g(104+81 g) \\
& +5 h(597+997 h))) \text {, } \\
& \psi_{4,5}=\frac{512}{15}\left(720 d^{5}(e-h)+d^{2}\left(e(-24221+3900 e)+6 g(231+100 g)+8555 h+9780 h^{2}\right)\right. \\
& +f\left(6 e^{2}(231+100 f)-3 e(1825+862 f)+g(908+686 g+f(881+540 g+20 f(19+20 g\right. \\
& +6 f(10+6 f+3 g))))-5(499+f(499+4 f(115+12 f(8+3 f)))) h+10(499 \\
& \left.+2 f(153+2 f(38+9 f))) h^{2}\right)-d\left(-21022 e+4406 e^{2}+3 g(1825+862 g)+8555 h(-1+2 h)\right) \\
& \left.+120 d^{4}(e(-46+3 e)+h(40+3 h))-20 d^{3}(e(-803+92 e)+h(563+148 h))\right), \\
& \psi_{4,6}=\frac{512}{3}\left(48 d^{5}(-e+h)+f\left(-4 e^{2}(151+38 f)+e(3109+908 f)+g(-913-380 g-4 f(173\right.\right. \\
& +58 g+3 f(41+10 g+2 f(15+2 f+g))))+12\left(101+f\left(101+f\left(75+34 f+4 f^{2}\right)\right)\right) h \\
& \left.-12(202+f(87+2 f(12+f))) h^{2}\right)-24 d^{4}((-27+e) e+h(25+h))+d(e(-8053+1300 e)
\end{aligned}
$$




$$
\begin{aligned}
& +g(3109+908 g)+2736 h(-1+2 h))-4 d^{2}(2 e(-785+92 e)+g(151+38 g) \\
& \left.+171 h(4+3 h))+12 d^{3}(3 e(-79+6 e)+h(187+32 h))\right), \\
\psi_{4,7}= & -2048\left(12 d^{4}(e-h)+f\left(-2 e^{2}(29+4 f)+e(412+74 f)-g(183+44 g+f(107\right.\right. \\
& \left.+18 g+4 f(13+3 f+g)))+(127+f(127+4 f(16+3 f))) h-2\left(127+34 f+4 f^{2}\right) h^{2}\right) \\
& +4 d^{3}((-27+e) e+2 h(12+h))+d\left(e(-785+92 e)+412 g+74 g^{2}+207 h(-1+2 h)\right) \\
& \left.-d^{2}\left(5 e(-79+6 e)+58 g+8 g^{2}+23 h(9+4 h)\right)\right), \\
\psi_{4,8}= & -2048\left(20 d^{3}(e-h)+f\left(2 e^{2}(17+f)-e(373+38 f)+g\left(229+90 f+20 f^{2}\right.\right.\right. \\
& \left.+6(5+f) g)-20(2+f)^{2} h+20(8+f) h^{2}\right)+2 d^{2}(3(-27+e) e+g(17+g)+10 h(5+h)) \\
& -d(7 e(-79+6 e)+g(373+38 g)+100 h(-1+2 h))), \\
\psi_{4,9}= & -8192\left(d^{2}(7 e-2 g-5 h)+f\left(-2 e^{2}+e(45+2 f)-g(36+7 f+2 g)+5(1+f) h-10 h^{2}\right)\right. \\
& +d(2(-27+e) e+g(45+2 g)+5 h(-1+2 h))), \\
\psi_{4,10}= & -73728(d-f)(e-g) .
\end{aligned}
$$

\section{A.4 Higher point function on $\mathcal{R}_{n}$}

As the $m(m \geq 5)$ point function in complex plane can be solved by using Ward identities recursively. The m point function on $\mathcal{R}_{n}$ can be transformed to a set of summations. The basic $\mathrm{k}$ summation function is

$$
S\left[\left\{a_{i j}\right\} ;\left\{r_{i j}\right\} ; 1 \leq i<j \leq m\right]=\sum_{j_{1}, \cdots, j_{m}=0}^{n-1} \prod_{1 \leq k<l \leq m} \sinh ^{-a_{k l}}\left(\frac{\log \sqrt{r_{k l}}}{n}+\frac{\pi i\left(j_{k}-j_{l}\right)}{n}\right) .
$$

This function is reduced to two,three and four point case when $m=2,3,4$. Though residue theorem can be used to compute it, it would become tedious quickly as $m$ increases. As an example, for four spin 3 point function on $\mathcal{R}_{n}$, the number of independent terms is expected to be $\mathcal{O}\left(10^{2}\right)$ as it is proportional to six point functions(four spin 3 and two twist operator). So for $m>4$, to find an explicit answer is horrible. It is interesting to study this summation in other methods, this would be important to read out the information of $\mu^{m}$ correction to Rényi and entanglement entropy.

\section{B Integrals}

All the integrals appeared in this work can be converted to a few types of basic integral labeled by two real number $p$ and $\mathrm{q}$,

$$
G[p, q ; x]=\int^{x} d x \log ^{p} x /(x-a)^{q} .
$$

In general, $\mathrm{p}$ and $\mathrm{q}$ can be real numbers. However, in this work $\mathrm{p}$ is always a non-negative integer and $\mathrm{q}$ is an integer. If $q \leq 0$, after doing integral by parts, we can find the answer. Also, if $p=0$, the answer is simple. So we only focus on positive integers $p, q$. Due to the recursion relation

$$
\frac{1}{(x-a)^{q}}=\frac{1}{q-1} \frac{d \frac{1}{(x-a)^{q-1}}}{d a}
$$


it is safe to study $q=1$. Actually to calculate the $\mathcal{O}\left(\mu^{k}\right)$ correction of HSEE, we only need the integrals $p \leq k-1$. In this work, $k \leq 4$, then there are three types of integrals.

1. $p=1$.

$$
G[1,1 ; x]=\log [x] \log [1-x / a]+\text { PolyLog }[2, x / a]
$$

The actual integral is from $0 \rightarrow \infty$, hence the limit behaviour $x \rightarrow 0$ and $x \rightarrow \infty$ of $G[p, q ; x]$ is important.

$$
\begin{aligned}
& \lim _{x \rightarrow 0} G[1,1 ; x]=0 \\
& \lim _{x \rightarrow \infty} G[1,1 ; x]=\frac{1}{6}\left(-\pi^{2}-3 \log \left[-\frac{1}{a}\right]^{2}+3 \log [1 / x]^{2}\right)
\end{aligned}
$$

For the terms $-\pi^{2}-3 \log \left[-\frac{1}{a}\right]^{2}$ in (B.5), it seems that we can set them to zero as this will lead to correct answer. We just throw them out by hand. Therefore we find a rule before taking the limit of $x \rightarrow \infty$,

$$
\text { PolyLog }[2, x / a] \rightarrow-\log [x] \log [1-x / a]+\frac{1}{2} \log [x]^{2}
$$

2. $p=2$.

$$
G[2,1 ; x]=\log [x]^{2} \log [1-x / a]+2 \log [x] \text { PolyLog }[2, x / a]-2 \text { PolyLog }[3, x / a]
$$

and the limit behaviour is

$$
\begin{aligned}
& \lim _{x \rightarrow 0} G[2,1 ; x]=0 \\
& \lim _{x \rightarrow \infty} G[2,1 ; x]=\frac{1}{3}\left(\pi^{2} \log \left[-\frac{1}{a}\right]+\log \left[-\frac{1}{a}\right]^{3}-\log [1 / x]^{3}\right)
\end{aligned}
$$

The same reasoning as $p=1$ case leads us to a replacement rule when $x \rightarrow \infty$,

$$
\text { PolyLog }[3, x / a] \rightarrow \frac{1}{3} \log [x]^{3}-\frac{1}{2} \log [x]^{2} \log [1-x / a]
$$

3. $p=3$,

$$
\begin{aligned}
G[3,1 ; x]= & \log [x]^{3} \log [1-x / a]+3 \log [x]^{2} \text { PolyLog }[2, x / a] \\
& -6 \log [x] \text { PolyLog }[3, x / a]+6 \text { PolyLog }[4, x / a]
\end{aligned}
$$

Then the replacement rule is

$$
\text { PolyLog }[4, x / a] \rightarrow \frac{1}{8} \log [x]^{4}-\frac{1}{6} \log [x]^{3} \log [1-x / a]
$$

The three replacement rules (B.6), (B.10)and (B.12) are all we need when we take the $x \rightarrow \infty$ limit. 


\section{B.1 Integral $F[J, j]$}

Here, we list the integral of $F[J, j]$ up to spin 6 .

1. $J=3$. Since $j=0$ term is canceled, there are only two integrals.

(a)

$$
F[3,1]=\frac{1}{12(U-1)^{6}} \sum_{i=0}^{2} c[3,1, i] \log ^{i}[U]
$$

with

$$
\begin{aligned}
& c[3,1,0]=-(-1+U)^{2}\left(1-14 U+U^{2}\right) \\
& c[3,1,1]=2\left(-1+8 U-8 U^{3}+U^{4}\right) \\
& c[3,1,2]=12 U^{2}
\end{aligned}
$$

(b)

$$
F[3,2]=\frac{1}{2(U-1)^{8}} \sum_{i=0}^{2} c[3,2, i] \log ^{i}[U]
$$

with

$$
\begin{aligned}
& c[3,2,0]=(-1+U)^{2}\left(5+26 U+5 U^{2}\right) \\
& c[3,2,1]=-2\left(-1-16 U+16 U^{3}+U^{4}\right) \\
& c[3,2,2]=4 U\left(2+5 U+2 U^{2}\right)
\end{aligned}
$$

2. $J=4, j=1,2,3$.

(a)

$$
F[4,1]=\frac{1}{180(-1+U)^{8}} \sum_{i=0}^{2} c[4,1, i] \log ^{i}[U]
$$

with

$$
\begin{aligned}
& c[4,1,0]=(-1+U)^{2}\left(2-23 U+222 U^{2}-23 U^{3}+2 U^{4}\right) \\
& c[4,1,1]=-6\left(-1+9 U-45 U^{2}+45 U^{4}-9 U^{5}+U^{6}\right) \\
& c[4,1,2]=180 U^{3}
\end{aligned}
$$

(b)

$$
F[4,2]=\frac{1}{36(-1+U)^{10}} \sum_{i=0}^{2} c[4,2, i] \log ^{i}[U]
$$

with

$$
\begin{aligned}
& c[4,2,0]=-(-1+U)^{2}\left(11-248 U-966 U^{2}-248 U^{3}+11 U^{4}\right) \\
& c[4,2,1]=6\left(-1+18 U+207 U^{2}-207 U^{4}-18 U^{5}+U^{6}\right) \\
& c[4,2,2]=36 U^{2}\left(9+22 U+9 U^{2}\right)
\end{aligned}
$$


(c)

$$
F[4,3]=\frac{1}{3(-1+U)^{12}} \sum_{i=0}^{2} c[4,3, i] \log ^{i}[U]
$$

with

$$
\begin{aligned}
& c[4,3,0]=(-1+U)^{2}\left(10+209 U+462 U^{2}+209 U^{3}+10 U^{4}\right) \\
& c[4,3,1]=-3\left(-1-54 U-189 U^{2}+189 U^{4}+54 U^{5}+U^{6}\right) \\
& c[4,3,2]=9 U\left(3+24 U+46 U^{2}+24 U^{3}+3 U^{4}\right)
\end{aligned}
$$

3. $J=5 ; j=1,2,3,4$.

(a)

$$
F[5,1]=\frac{1}{5040(-1+U)^{10}} \sum_{i=0}^{2} c[5,1, i] \log ^{i}[U]
$$

with

$$
\begin{aligned}
& c[5,1,0]=-(-1+U)^{2}\left(9-110 U+779 U^{2}-6396 U^{3}+779 U^{4}-110 U^{5}+9 U^{6}\right) \\
& c[5,1,1]=12\left(-3+32 U-168 U^{2}+672 U^{3}-672 U^{5}+168 U^{6}-32 U^{7}+3 U^{8}\right) \\
& c[5,1,2]=5040 U^{4}
\end{aligned}
$$

(b)

$$
F[5,2]=\frac{1}{360(-1+U)^{12}} \sum_{i=0}^{2} c[5,2, i] \log ^{i}[U]
$$

with

$$
\begin{gathered}
c[5,2,0]=(-1+U)^{2}\left(19-314 U+4745 U^{2}+16300 U^{3}\right. \\
\left.+4745 U^{4}-314 U^{5}+19 U^{6}\right) \\
c[5,2,1]=-12\left(-1+16 U-184 U^{2}-1776 U^{3}+1776 U^{5}\right. \\
\left.+184 U^{6}-16 U^{7}+U^{8}\right) \\
c[5,2,2]=720 U^{3}\left(8+19 U+8 U^{2}\right)
\end{gathered}
$$

(c)

$$
F[5,3]=\frac{1}{72(-1+U)^{14}} \sum_{i=0}^{2} c[5,3, i] \log ^{i}[U]
$$

with

$$
\begin{aligned}
& c[5,3,0]=-(-1+U)^{2}\left(31-1218 U-17907 U^{2}\right. \\
&\left.-37412 U^{3}-17907 U^{4}-1218 U^{5}+31 U^{6}\right) \\
& c[5,3,1]=12\left(-1+32 U+1232 U^{2}+\right. 3744 U^{3} \\
&\left.-3744 U^{5}-1232 U^{6}-32 U^{7}+U^{8}\right) \\
& c[5,3,2]=144 U^{2}\left(18+128 U+233 U^{2}+128 U^{3}+18 U^{4}\right)
\end{aligned}
$$


(d)

$$
F[5,4]=\frac{1}{36(-1+U)^{16}} \sum_{i=0}^{2} c[5,4, i] \log ^{i}[U]
$$

with

$$
\begin{aligned}
c[5,4,0]=(-1+U)^{2}(141+6874 U & +42935 U^{2} \\
& \left.+76500 U^{3}+42935 U^{4}+6874 U^{5}+141 U^{6}\right) \\
c[5,4,1]=-12\left(-3-352 U-3312 U^{2}-7008 U^{3}\right. & \left.+7008 U^{5}+3312 U^{6}+352 U^{7}+3 U^{8}\right) \\
c[5,4,2]=144 U\left(4+66 U+300 U^{2}+\right. & \left.485 U^{3}+300 U^{4}+66 U^{5}+4 U^{6}\right)
\end{aligned}
$$

4. $J=6 ; j=1,2,3,4,5$.

(a)

$$
F[6,1]=\frac{1}{25200(-1+U)^{12}} \sum_{i=0}^{2} c[6,1, i] \log ^{i}[U]
$$

with

$$
\begin{gathered}
c[6,1,0]=(-1+U)^{2}\left(8-109 U+774 U^{2}-4343 U^{3}+32540 U^{4}\right. \\
\left.-4343 U^{5}+774 U^{6}-109 U^{7}+8 U^{8}\right) \\
c[6,1,1]=-20\left(-2+25 U-150 U^{2}+600 U^{3}-2100 U^{4}+2100 U^{6}\right. \\
c[6,1,2]=25200 U^{5}
\end{gathered}
$$

(b)

$$
F[6,2]=\frac{1}{25200(-1+U)^{14}} \sum_{i=0}^{2} c[6,2, i] \log ^{i}[U]
$$

with

$$
\begin{gathered}
c[6,2,0]=-(-1+U)^{2}\left(261-4228 U+42408 U^{2}-538956 U^{3}-1720570 U^{4}\right. \\
\left.-538956 U^{5}+42408 U^{6}-4228 U^{7}+261 U^{8}\right) \\
c[6,2,1]=60\left(-3+50 U-475 U^{2}+4400 U^{3}+37800 U^{4}-37800 U^{6}\right. \\
\left.-4400 U^{7}+475 U^{8}-50 U^{9}+3 U^{10}\right) \\
c[6,2,2]=25200 U^{4}\left(25+58 U+25 U^{2}\right)
\end{gathered}
$$

(c)

$$
F[6,3]=\frac{1}{1800(-1+U)^{16}} \sum_{i=0}^{2} c[6,3, i] \log ^{i}[U]
$$

with

$$
\begin{aligned}
c[6,3,0]=(-1+U)^{2}(137- & 3601 U+94286 U^{2}+1146173 U^{3}+2288810 U^{4} \\
+ & \left.1146173 U^{5}+94286 U^{6}-3601 U^{7}+137 U^{8}\right)
\end{aligned}
$$




$$
\begin{gathered}
c[6,3,1]=-60\left(-1+25 U-525 U^{2}-16400 U^{3}-45100 U^{4}+45100 U^{6}\right. \\
\left.+16400 U^{7}+525 U^{8}-25 U^{9}+U^{10}\right) \\
c[6,3,2]=3600 U^{3}\left(50+325 U+573 U^{2}+325 U^{3}+50 U^{4}\right)
\end{gathered}
$$

(d)

$$
F[6,4]=\frac{1}{360(-1+U)^{18}} \sum_{i=0}^{2} c[6,4, i] \log ^{i}[U]
$$

with

$$
\begin{gathered}
c[6,4,0]=-(-1+U)^{2}\left(187-11376 U-382064 U^{2}-2076752 U^{3}-3527190 U^{4}\right. \\
\left.-2076752 U^{5}-382064 U^{6}-11376 U^{7}+187 U^{8}\right) \\
c[6,4,1]=60\left(-1+50 U+4175 U^{2}+32800 U^{3}+62800 U^{4}-62800 U^{6}\right. \\
\left.-32800 U^{7}-4175 U^{8}-50 U^{9}+U^{10}\right) \\
c[6,4,2]=3600 U^{2}\left(10+140 U+575 U^{2}+902 U^{3}+575 U^{4}+140 U^{5}+10 U^{6}\right)
\end{gathered}
$$

(e)

$$
F[6,5]=\frac{1}{180(-1+U)^{20}} \sum_{i=0}^{2} c[6,5, i] \log ^{i}[U]
$$

with

$$
\begin{gathered}
c[6,5,0]=(-1+U)^{2}\left(786+70697 U+873858 U^{2}+3485019 U^{3}+5427680 U^{4}\right. \\
\left.+3485019 U^{5}+873858 U^{6}+70697 U^{7}+786 U^{8}\right) \\
c[6,5,1]=-60(-3-625 U- \\
11125 U^{2}-56500 U^{3}-89250 U^{4}+89250 U^{6} \\
\left.+56500 U^{7}+11125 U^{8}+625 U^{9}+3 U^{10}\right) \\
c[6,5,2]=900 U\left(5+140 U+1160 U^{2}+3820 U^{3}+5626 U^{4}+3820 U^{5}\right. \\
\left.+1160 U^{6}+140 U^{7}+5 U^{8}\right)
\end{gathered}
$$

\section{B.2 Integral in $\mathcal{O}\left(\mu^{4}\right)$}

The basic integral is

$$
\left(\prod_{k=1}^{4} \int d t_{k} f\left[J, t_{k}\right]\right) y_{12}^{a} y_{i 3}^{b} y_{j 4}^{c} .
$$

In this work, $a, b, c$ are positive integers and $1 \leq i \leq 2,1 \leq j \leq 3$. It is convenient to define a set of integrals as ${ }^{22}$

$$
\begin{aligned}
& m_{a}[t]=\int_{0}^{\infty} d t^{\prime} f\left[t^{\prime}\right] y\left[t, t^{\prime}\right]^{a} \\
& g_{a b}[t]=\int_{0}^{\infty} d t^{\prime} f\left[t^{\prime}\right] m_{b}\left[t^{\prime}\right] y\left[t, t^{\prime}\right]^{a}
\end{aligned}
$$

\footnotetext{
${ }^{22}$ We omit the dependence of $\mathrm{J}$ to simplify notation. In the following, our calculation is done for $J=3$.
} 


$$
\begin{aligned}
& k_{a b c}[t]=\int_{0}^{\infty} d t^{\prime} f\left[t^{\prime}\right] m_{b}\left[t^{\prime}\right] m_{c}\left[t^{\prime}\right] y\left[t, t^{\prime}\right]^{a} \\
& l_{a b c}[g]=\int_{0}^{\infty} d t^{\prime} f\left[t^{\prime}\right] g_{b c}\left[t^{\prime}\right] y\left[t, t^{\prime}\right]^{a} .
\end{aligned}
$$

Then after integrating out $t_{4}, t_{3}, t_{2}$, the terms $y_{12}^{a} y_{i 3}^{b} y_{j 4}^{c}$ are replaced by

$$
\begin{aligned}
y_{12}^{a} y_{13}^{b} y_{14}^{c} & \rightarrow m_{a}\left[t_{1}\right] m_{b}\left[t_{1}\right] m_{c}\left[t_{1}\right] \\
y_{12}^{a} y_{23}^{b} y_{14}^{c} & \rightarrow g_{a b}\left[t_{1}\right] m_{c}\left[t_{1}\right] \\
y_{12}^{a} y_{13}^{b} y_{24}^{c} & \rightarrow g_{a c}\left[t_{1}\right] m_{b}\left[t_{1}\right] \\
y_{12}^{a} y_{23}^{b} y_{24}^{c} & \rightarrow k_{a b c}\left[t_{1}\right] \\
y_{12}^{a} y_{13}^{b} y_{34}^{c} & \rightarrow m_{a}\left[t_{1}\right] g_{b c}\left[t_{1}\right] \\
y_{12}^{a} y_{23}^{b} y_{34}^{c} & \rightarrow l_{a b c}\left[t_{1}\right] .
\end{aligned}
$$

So the building blocks are these defined functions. One can integrate out them term by term, though very tedious. In our computation, we use a slightly different method. Instead of integrating out $t_{4}, t_{3}, t_{2}$ term by term, we just integrate out $t_{4}, t_{3}$ at first. And then we sum over the results. After that, the integral $t_{2}, t_{1}$ are done. That means we just do the following replacement,

$$
\begin{aligned}
y_{13}^{b} y_{14}^{c} & \rightarrow m_{b}\left[t_{1}\right] m_{c}\left[t_{1}\right] \\
y_{23}^{b} y_{14}^{c} & \rightarrow m_{b}\left[t_{2}\right] m_{c}\left[t_{1}\right] \\
y_{13}^{b} y_{24}^{c} & \rightarrow m_{b}\left[t_{1}\right] m_{c}\left[t_{2}\right] \\
y_{23}^{b} y_{24}^{c} & \rightarrow m_{b}\left[t_{2}\right] m_{c}\left[t_{2}\right] \\
y_{13}^{b} y_{34}^{c} & \rightarrow g_{b c}\left[t_{2}\right] \\
y_{23}^{b} y_{34}^{c} & \rightarrow g_{b c}\left[t_{2}\right] .
\end{aligned}
$$

The relevant $m_{a}$ s are the ones with $a=1,2, \cdots, 5$, and the relevant $g_{b c}$ are

$$
b+c \leq 6, b \geq 1, c \geq 1
$$

So the number of integrals we need to do is $(5+5+4+3+2+1)=20$.

Then we classify the function according to the power of $n$ and $y_{12}$. A general function is

$$
n^{2 \alpha+1} y_{12}^{a} \mathcal{F}_{\alpha, a}\left(t_{1}, t_{2}\right) / n^{8}
$$

where $\mathcal{F}_{\alpha, a}\left(t_{1}, t_{2}\right)$ is determined by $m_{a}, g_{a b} . \alpha, a$ satisfy

$$
1 \leq \alpha \leq 4,1 \leq a \leq 2 \alpha+2
$$

So there are $(4+6+8+10=) 28$ different integrals of $t_{2}$ in (B.29). In the work, we calculate the $\mathcal{O}(c)$ part and the quantum correction, there is no divergence after we sum over the integrals of $t_{4}, t_{3}, t_{2}$.

After all these have been done, we need to integrate $t_{1}$. We can integrate it according to the power of $n$. So there are 4 integrals of $t_{1}$. Since the Rényi entropy at this order 
should be finite, the integration should cancel the divergent term in the partition function $n \log Z_{1}$. So the divergence should come from $\alpha=4$ terms. For $\alpha=4$, we should be careful to seperate the divergence term. There is no other subtlety except these. In total, we need to do $(28+20+4=) 52$ integrals. We just mention that the first 28 and the last 4 integrals is relatively simple, whilst the other 20 integrals of $t_{2}$ is a bit complicated. As an illustration, we just give the results of $m_{1}[t]$, the other functions $m_{a}[t], g_{a b}[t]$ are similar.

$$
\begin{aligned}
m_{1}[t]=\frac{1}{-1+U}(-1+t)\left(-\frac{1}{2(-1+t)(-1+U)^{2}}-\frac{U}{(-1+U)^{3}(-t+U)}\right. \\
-\frac{1+U-2 t U}{(-1+t)^{2}(-1+U)^{3}}-\frac{t^{2} \log [t]}{(-1+t)^{3}(t-U)^{2}} \\
\left.+\frac{U(-t(2+U)+U(1+2 U)) \log [U]}{(t-U)^{2}(-1+U)^{4}}\right) .
\end{aligned}
$$

\section{C $\mathcal{O}\left(\mu^{2}\right)$ correction to Rényi entropy and entanglement entropy of other spins}

The formula for Rényi entropy to $\mathcal{O}\left(\mu_{J}^{2}\right)$ is (6.7). One can plug the value of $\tilde{b}[J, j ; n]$ and $F[J, j]$ to find their explicit expression. Taking the limit $n \rightarrow 1$, we can list the $\mathcal{O}\left(\mu_{J}^{2}\right)$ corrections of entanglement entropies from spin 4 to spin 6 below,

$$
\begin{aligned}
\left.S\right|_{\text {spin } 4, \mu_{4}^{2}} & =\frac{8 \mu_{4}^{2} \mathcal{N}_{4} \pi^{6}}{105 \beta^{4}(U-1)^{6}} \sum_{i=0}^{2} \kappa[4, i] \log ^{i}[U], \\
\left.S\right|_{\text {spin } 5, \mu_{5}^{2}} & =-\frac{32 \mu_{5}^{2} \mathcal{N}_{5} \pi^{8}}{2835 \beta^{6}(U-1)^{8}} \sum_{i=0}^{2} \kappa[5, i] \log ^{i}[U] \\
\left.S\right|_{\text {spin } 6, \mu_{6}^{2}} & =\frac{64 \mu_{6}^{2} \mathcal{N}_{6} \pi^{10}}{10395 \beta^{8}(U-1)^{10}} \sum_{i=0}^{2} \kappa[6, i] \log ^{i}[U]
\end{aligned}
$$

with

$$
\begin{gathered}
\kappa[4,0]=-5\left(5+27 U^{2}-64 U^{3}+27 U^{4}+5 U^{6}\right) \\
\kappa[4,1]=6\left(-3-8 U-35 U^{2}+35 U^{4}+8 U^{5}+3 U^{6}\right) \\
\kappa[4,2]=-36 U\left(1+U+6 U^{2}+U^{3}+U^{4}\right) \\
\kappa[5,0]=-(-1+U)^{2}\left(213+982 U+7175 U^{2}+8460 U^{3}\right. \\
\left.\quad+7175 U^{4}+982 U^{5}+213 U^{6}\right) \\
\kappa[5,1]=24\left(-6-29 U-279 U^{2}-381 U^{3}+381 U^{5}+279 U^{6}+29 U^{7}+6 U^{8}\right) \\
\kappa[5,2]=-360 U\left(1+3 U+21 U^{2}+20 U^{3}+21 U^{4}+3 U^{5}+U^{6}\right) \\
\kappa[6,0]=-(-1+U)^{2}\left(463+3751 U+43414 U^{2}+119077 U^{3}+195790 U^{4}\right. \\
\left.\quad+119077 U^{5}+43414 U^{6}+3751 U^{7}+463 U^{8}\right) \\
\kappa[6,1]=60\left(-5-37 U-597 U^{2}-1972 U^{3}-2912 U^{4}+2912 U^{6}\right. \\
\left.\quad+1972 U^{7}+597 U^{8}+37 U^{9}+5 U^{10}\right)
\end{gathered}
$$




$$
\begin{aligned}
\kappa[6,2]=-900 U\left(1+6 U+56 U^{2}+\right. & 126 U^{3}+210 U^{4} 6 \\
+ & \left.126 U^{5}+56 U^{6}+6 U^{7}+U^{8}\right)
\end{aligned}
$$

\section{Quantum correction of partition function of higher spin black hole}

In this appendix, we use the zero mode insertion method [39] to calculate the quantum correction of the partition function of spin 3 black hole. We will find the same result as section 5. In their method, the partition function is supposed to be $\mathrm{b}^{23}$

$$
Z_{C F T}(\hat{\tau}, \alpha)=\operatorname{Tr} \hat{q}^{L_{0}-\frac{c}{24}} y^{W_{0}}, \hat{q}=e^{2 \pi i \hat{\tau}}, y=e^{2 \pi i \alpha}
$$

In the perturbation theory, the partition function is expanded by the power of $\alpha$,

$$
Z_{C F T}(\hat{\tau}, \alpha)=\operatorname{Tr}\left(\hat{q}^{L_{0}-\frac{c}{24}}\right)+\frac{(2 \pi i \alpha)^{2}}{2} \operatorname{Tr}\left(W_{0}^{2} \hat{q}^{L_{0}-\frac{c}{24}}\right)+\frac{(2 \pi i \alpha)^{4}}{4 !} \operatorname{Tr}\left(W_{0}^{4} \hat{q}^{L_{0}-\frac{c}{24}}\right)+\cdots
$$

In the high temperature regime, after a $\mathrm{S}$ modular transformation, the trace is contributed by vacuum state. There is no quantum correction at order $\alpha^{2}$, hence we proceed to the $\alpha^{4}$ correction. Borrowing the notation in [39],

$$
Z^{(4)}=\frac{\alpha^{4} \tau^{8}}{4 !}\left(3 I_{1}\left(A_{1}+A_{2}\right)+3 I_{2} A_{6}+\frac{5}{2} I_{3} A_{3}+\frac{5}{3} I_{4} A_{4}+I_{5} A_{5}\right)
$$

As they ignore the subleading order in $1 / \mathrm{c}$, we denote their result (3.19) and (3.20) by $A_{i}^{c l}$. Schematically, we can write

$$
A_{i}=A_{i}^{c l}+A_{i}^{q u}
$$

To read out the quantum correction, we consider the effect from the composite operator $\Lambda=: T T:(z)-\frac{3}{10} \partial^{2} T(z)$. In their convention, it is easy to find

$$
\left[\Lambda_{m}, \Lambda_{n}\right]=\frac{c(5 c+22)}{10 \times 7 !} m\left(m^{2}-1\right)\left(m^{2}-4\right)\left(m^{2}-9\right) \delta_{m,-n}+\cdots
$$

The $\cdots$ term has no contribution at order $\alpha^{4}$. We define some notation,

$$
\begin{aligned}
& W[0]=(2 \pi i)^{-1} \sum_{j \geq-2} a_{j} W_{j} \\
& W[1]=(2 \pi i)^{-2} \sum_{j \geq-1} b_{j} W_{j} \\
& W[2]=(2 \pi i)^{-3} \sum_{j \geq 0} c_{j} W_{j} \\
& W[3]=(2 \pi i)^{-4} \sum_{j \geq 1} d_{j} W_{j}
\end{aligned}
$$

\footnotetext{
${ }^{23} \operatorname{In}[39], \hat{\tau}$ is the parameter $\tau$ in this paper. Only in this appendix, we change our convention to match those in [39]. The parameter $\tau$ in this appendix is $\tau=-\frac{1}{\tilde{\tau}}$.
} 
The values of $a_{j}, b_{j}, c_{j}, d_{j}$ can be read from [39], appendix B.1. We use $A_{1}$ as an example.

$$
\begin{aligned}
A_{1}=b_{5} b_{-1} b_{-1}\langle & \left.W_{5} W_{-1} W_{-1} W_{-3}\right\rangle+b_{4} b_{0} b_{-1}\left\langle W_{4} W_{0} W_{-1} W_{-3}\right\rangle \\
& +b_{4} b_{-1} b_{0}\left\langle W_{4} W_{-1} W_{0} W_{-3}\right\rangle+b_{3} b_{0} b_{0}\left\langle W_{3} W_{0} W_{0} W_{-3}\right\rangle \\
& +b_{3} b_{-1} b_{1}\left\langle W_{3} W_{-1} W_{1} W_{3}\right\rangle+b_{3} b_{1} b_{-1}\left\langle W_{3} W_{1} W_{-1} W_{-3}\right\rangle
\end{aligned}
$$

We note that in each term above, it is actually the vacuum expectation value of two commutator, for example

$$
\left\langle W_{5} W_{-1} W_{-1} W_{-3}\right\rangle=\left\langle\left[W_{5}, W_{-1}\right]\left[W_{-1}, W_{-3}\right]\right\rangle
$$

Using the nonlinear $W_{\infty}(\lambda)$ algebra

$$
\left[W_{m}, W_{n}\right] \sim U_{m+n}+L_{m+n}+\delta_{m,-n}+\frac{40 N_{3}}{5 c+22}(m-n) \Lambda_{m+n},
$$

The first three terms in the right hand side contribute $A_{i}^{c l}$, whist the last term contributes $A_{i}^{q u}$. We find

$$
A_{1}^{q u}=-\frac{416 c N_{3}^{2}}{63(22+5 c)}=A_{5}^{q u}, A_{2}^{q u}=-\frac{32 c N_{3}^{2}}{7(22+5 c)}=A_{3}^{q u}, A_{4}^{q u}=-A_{6}^{q u}=3 / 2 A_{2}^{q u} .
$$

Substituting the values of $I_{i}, N_{3}, a_{j}, b_{j}, c_{j}, d_{j}$ in that paper, we find the quantum correction of partition function at $\alpha^{4}$,

$$
\left.\log Z\right|_{\mu^{4}, \text { quan }}=\frac{640 i \alpha^{4} c \pi}{27(22+5 c) \hat{\tau}^{9}}
$$

Note (D.11) is exactly the same as (5.11).

Open Access. This article is distributed under the terms of the Creative Commons Attribution License (CC-BY 4.0), which permits any use, distribution and reproduction in any medium, provided the original author(s) and source are credited.

\section{References}

[1] C. Holzhey, F. Larsen and F. Wilczek, Geometric and renormalized entropy in conformal field theory, Nucl. Phys. B 424 (1994) 443 [hep-th/9403108] [InSPIRE].

[2] P. Calabrese and J.L. Cardy, Entanglement entropy and quantum field theory, J. Stat. Mech. 0406 (2004) P06002 [hep-th/0405152] [INSPIRE].

[3] P. Calabrese and J.L. Cardy, Evolution of entanglement entropy in one-dimensional systems, J. Stat. Mech. 0504 (2005) P04010 [cond-mat/0503393] [INSPIRE].

[4] A. Kitaev and J. Preskill, Topological entanglement entropy, Phys. Rev. Lett. 96 (2006) 110404 [hep-th/0510092] [INSPIRE].

[5] M. Levin and X.G. Wen, Detecting topological order in a ground state wave function, Phys. Rev. Lett. 96 (2006) 110405 [cond-mat/0510613].

[6] B. Sundborg, Stringy gravity, interacting tensionless strings and massless higher spins, Nucl. Phys. Proc. Suppl. 102 (2001) 113 [hep-th/0103247] [inSPIRE]. 
[7] A. Mikhailov, Notes on higher spin symmetries, hep-th/0201019 [INSPIRE].

[8] I.R. Klebanov and A.M. Polyakov, AdS dual of the critical $\mathrm{O}(N)$ vector model, Phys. Lett. B 550 (2002) 213 [hep-th/0210114] [INSPIRE].

[9] M.A. Vasiliev, Consistent equation for interacting gauge fields of all spins in (3+1)-dimensions, Phys. Lett. B 243 (1990) 378 [InSPIRE].

[10] M.A. Vasiliev, More on equations of motion for interacting massless fields of all spins in (3+1)-dimensions, Phys. Lett. B 285 (1992) 225 [inSPIRE].

[11] M.R. Gaberdiel and R. Gopakumar, An AdS 3 dual for minimal model CFTs, Phys. Rev. D 83 (2011) 066007 [arXiv: 1011.2986] [INSPIRE].

[12] S.F. Prokushkin and M.A. Vasiliev, Higher spin gauge interactions for massive matter fields in 3D AdS space-time, Nucl. Phys. B 545 (1999) 385 [hep-th/9806236] [INSPIRE].

[13] M.R. Gaberdiel and R. Gopakumar, Minimal model holography, J. Phys. A 46 (2013) 214002 [arXiv: 1207.6697 ] [INSPIRE].

[14] M. Ammon, M. Gutperle, P. Kraus and E. Perlmutter, Black holes in three dimensional higher spin gravity: a review, J. Phys. A 46 (2013) 214001 [arXiv:1208.5182] [INSPIRE].

[15] B. Chen, J. Long and J.-j. Zhang, Holographic Rényi entropy for CFT with W symmetry, JHEP 04 (2014) 041 [arXiv: 1312.5510] [INSPIRE].

[16] E. Perlmutter, Comments on Rényi entropy in $A d S_{3} / C F T_{2}$, JHEP 05 (2014) 052 [arXiv:1312.5740] [INSPIRE].

[17] A. Achucarro and P.K. Townsend, A Chern-Simons action for three-dimensional Anti-de Sitter supergravity theories, Phys. Lett. B 180 (1986) 89 [InSPIRE].

[18] E. Witten, (2+1)-dimensional gravity as an exactly soluble system, Nucl. Phys. B 311 (1988) 46 [INSPIRE].

[19] M.P. Blencowe, A consistent interacting massless higher spin field theory in $D=(2+1)$, Class. Quant. Grav. 6 (1989) 443 [inSPIRE].

[20] M. Henneaux and S.-J. Rey, Nonlinear $W_{\infty}$ as asymptotic symmetry of three-dimensional higher spin Anti-de Sitter gravity, JHEP 12 (2010) 007 [arXiv: 1008.4579] [INSPIRE].

[21] A. Campoleoni, S. Fredenhagen, S. Pfenninger and S. Theisen, Asymptotic symmetries of three-dimensional gravity coupled to higher-spin fields, JHEP 11 (2010) 007 [arXiv: 1008.4744] [INSPIRE].

[22] M.R. Gaberdiel and R. Gopakumar, Triality in minimal model holography, JHEP 07 (2012) 127 [arXiv: 1205.2472] [INSPIRE].

[23] M. Gutperle and P. Kraus, Higher spin black holes, JHEP 05 (2011) 022 [arXiv:1103.4304] [INSPIRE].

[24] M. Ammon, P. Kraus and E. Perlmutter, Scalar fields and three-point functions in D $=3$ higher spin gravity, JHEP 07 (2012) 113 [arXiv:1111.3926] [INSPIRE].

[25] P. Kraus and E. Perlmutter, Probing higher spin black holes, JHEP 02 (2013) 096 [arXiv: 1209.4937] [INSPIRE].

[26] B. Chen, J. Long and Y.-N. Wang, Phase structure of higher spin black hole, JHEP 03 (2013) 017 [arXiv:1212.6593] [INSPIRE]. 
[27] L. Smarr, Mass formula for Kerr black holes, Phys. Rev. Lett. 30 (1973) 71 [Erratum ibid. 30 (1973) 521-521] [INSPIRE].

[28] M. Bañados, R. Canto and S. Theisen, The action for higher spin black holes in three dimensions, JHEP 07 (2012) 147 [arXiv:1204.5105] [INSPIRE].

[29] J.R. David, M. Ferlaino and S.P. Kumar, Thermodynamics of higher spin black holes in 3D, JHEP 11 (2012) 135 [arXiv:1210.0284] [INSPIRE].

[30] J. de Boer and J.I. Jottar, Thermodynamics of higher spin black holes in AdS $S_{3}$, JHEP 01 (2014) 023 [arXiv: 1302.0816] [INSPIRE].

[31] G.W. Gibbons and S.W. Hawking, Action integrals and partition functions in quantum gravity, Phys. Rev. D 15 (1977) 2752 [inSPIRE].

[32] M. Ammon, A. Castro and N. Iqbal, Wilson lines and entanglement entropy in higher spin gravity, JHEP 10 (2013) 110 [arXiv: 1306.4338] [INSPIRE].

[33] E. Hijano and P. Kraus, A new spin on entanglement entropy, arXiv:1406.1804 [INSPIRE].

[34] R.M. Wald, Black hole entropy is Noether charge, Phys. Rev. D 48 (1993) R3427 [gr-qc/9307038] [INSPIRE].

[35] P. Kraus and T. Ugajin, An entropy formula for higher spin black holes via conical singularities, JHEP 05 (2013) 160 [arXiv:1302.1583] [INSPIRE].

[36] S.N. Solodukhin, Entanglement entropy of black holes, Living Rev. Rel. 14 (2011) 8 [arXiv:1104.3712] [INSPIRE].

[37] D.V. Fursaev and S.N. Solodukhin, On the description of the Riemannian geometry in the presence of conical defects, Phys. Rev. D 52 (1995) 2133 [hep-th/9501127] [INSPIRE].

[38] P. Kraus and E. Perlmutter, Partition functions of higher spin black holes and their CFT duals, JHEP 11 (2011) 061 [arXiv:1108.2567] [INSPIRE].

[39] M.R. Gaberdiel, T. Hartman and K. Jin, Higher spin black holes from CFT, JHEP 04 (2012) 103 [arXiv: 1203.0015] [INSPIRE].

[40] M.R. Gaberdiel, K. Jin and E. Perlmutter, Probing higher spin black holes from CFT, JHEP 10 (2013) 045 [arXiv: 1307.2221] [INSPIRE].

[41] M. Beccaria and G. Macorini, On the partition functions of higher spin black holes, JHEP 12 (2013) 027 [arXiv:1310.4410] [INSPIRE].

[42] M. Beccaria and G. Macorini, Analysis of higher spin black holes with spin-4 chemical potential, JHEP 07 (2014) 047 [arXiv: 1312.5599] [INSPIRE].

[43] G. Compère, J.I. Jottar and W. Song, Observables and microscopic entropy of higher spin black holes, JHEP 11 (2013) 054 [arXiv:1308.2175] [INSPIRE].

[44] S. Datta, J.R. David, M. Ferlaino and S.P. Kumar, Higher spin entanglement entropy from CFT, JHEP 06 (2014) 096 [arXiv: 1402.0007] [INSPIRE].

[45] S. Datta, Relative entropy in higher spin holography, arXiv:1406.0520 [INSPIRE].

[46] S. Ryu and T. Takayanagi, Holographic derivation of entanglement entropy from AdS/CFT, Phys. Rev. Lett. 96 (2006) 181602 [hep-th/0603001] [INSPIRE].

[47] J. de Boer and J.I. Jottar, Entanglement entropy and higher spin holography in AdS $S_{3}, J H E P$ 04 (2014) 089 [arXiv: 1306.4347] [INSPIRE]. 
[48] S. Datta, J.R. David, M. Ferlaino and S.P. Kumar, A universal correction to higher spin entanglement entropy, Phys. Rev. D 90 (2014) 041903 [arXiv:1405.0015] [INSPIRE].

[49] P. Calabrese, J. Cardy and E. Tonni, Entanglement entropy of two disjoint intervals in conformal field theory, J. Stat. Mech. 0911 (2009) P11001 [arXiv: 0905.2069] [INSPIRE].

[50] P. Calabrese, J. Cardy and E. Tonni, Entanglement entropy of two disjoint intervals in conformal field theory II, J. Stat. Mech. 1101 (2011) P01021 [arXiv: 1011.5482] [INSPIRE].

[51] M. Headrick, Entanglement Rényi entropies in holographic theories, Phys. Rev. D 82 (2010) 126010 [arXiv: 1006.0047] [INSPIRE].

[52] B. Chen and J.-J. Zhang, On short interval expansion of Rényi entropy, JHEP 11 (2013) 164 [arXiv: 1309.5453] [INSPIRE]. 